\title{
Star formation in the bright rimmed globule IC 1396N
}

\author{
C. Codella ${ }^{1}$, R. Bachiller ${ }^{2}$, B. Nisini ${ }^{3}$, P. Saraceno ${ }^{1}$, and L. Testi ${ }^{4}$ \\ 1 Istituto di Fisica dello Spazio Interplanetario, CNR, Area di Ricerca Tor Vergata, Via Fosso del Cavaliere 100, \\ 00133 Roma, Italy \\ 2 Observatorio Astronómico Nacional (IGN), Apartado 1143, 28800 Alcalá de Henares (Madrid), Spain \\ 3 Osservatorio Astronomico di Roma, Via di Frascati 33, 00040 Monteporzio, Italy \\ 4 Osservatorio Astrofisico di Arcetri, Largo E. Fermi 5, 50125 Firenze, Italy
}

Received 13 February 2001 / Accepted 2 July 2001

\begin{abstract}
We report mm-wave multiline and continuum observations of IC 1396N, a conspicuous bright, rimmed globule excited by the O6.5 star HD 206267 in the Cep OB2 association. Single-dish high resolution observations in CO and CS lines reveal the cometary structure of the globule with unprecedented detail. The globule head contains a dense core of $0.2 \mathrm{pc}$, whereas the tail, pointing away from the exciting star, has a total length of 0.8 pc. Two high velocity bipolar outflows have been identified in the CO maps: the first one is located around the position of a strong IRAS source in the head of the globule, and the second one, which was previously unknown, is located in the northern region. The outflows emerge from high density clumps which exhibit strong line emission of $\mathrm{CS}, \mathrm{HCO}^{+}$, and $\mathrm{DCO}^{+}$. Within these clumps, the sources driving the outflows have been identified thanks to mm-wave continuum observations. The globule head harbors two YSOs separated by about $10^{4} \mathrm{AU}$. SiO line observations of the central outflow unveals a highly collimated structure with four clumps of sizes $\leq 0.1 \mathrm{pc}$, which are located along the outflow axis and suggest episodic events in the mass loss process from the central star. Kinetic temperatures of $\sim 50-100 \mathrm{~K}$ and hydrogen densities of fews $10^{6} \mathrm{~cm}^{-3}$ have been estimated in the shocked regions traced by the strong $\mathrm{SiO}$ emission. The jet is also exposed to view by the means of interferometric $\mathrm{HCO}^{+}$ observations that confirms that it is very narrow $(\leq 0.02 \mathrm{pc}$ wide). The detection of blue- and redshifted $\mathrm{CO}$ emission along the globule rim suggests that IC $1396 \mathrm{~N}$ is in a transient phase, undergoing one of the expansions or compressions predicted by theoretical models describing the evolution of cometary globules. Moreover, the CO data, together with near IR observations reported elsewhere, indicate that the star forming process is occurring also in the northern part of IC $1396 \mathrm{~N}$, at $0.5 \mathrm{pc}$ from the central CS peak. The present observations provide evidence that several star-forming sites can develop even in a moderately massive globule like IC 1396N.
\end{abstract}

Key words. ISM: clouds - ISM: individual objects: IC 1396N - ISM: jets and outflows - ISM: molecules - radio lines: ISM

\section{Introduction}

IC 1396 is a well-known extended H II region (S131, Sharpless 1959) located near the Cep OB2 association, at a distance of about $750 \mathrm{pc}$ from the Sun (Matthews 1979). The region is ionized by the O6.5 star HD 206267 , the brightest member of the young cluster Trumpler 37, which contains in addition about 20 B stars. The overall IC 1396 region has been extensively studied in the past (e.g. Weikard et al. 1996, and references therein), and several dusty globules were identified through $\mathrm{H} \alpha$ images (Osterbrock 1957), some of which have bright rims facing the central exciting star. At a projected distance of $\sim 11 \mathrm{pc}$ from HD 206267, near the border of the H II region (see

Send offprint requests to: C. Codella,

e-mail: codella@ifsi.rm.cnr.it
Fig. 2 of Weikard et al. 1996), IC $1396 \mathrm{~N}$ is one of the most conspicuous of such bright rimmed globules. It presents a striking cometary structure, and contains the very red object IRAS $21391+5802$, some strong $\mathrm{H}_{2} \mathrm{O}$ maser sources (Tofani et al. 1995; Slysh et al. 1999), and an extended bipolar molecular outflow (Sugitani et al. 1989). Thus the IC $1396 \mathrm{~N}$ globule is an active site of star formation.

The indication that stars can form in cometary globules has been already found for a limited number of targets (e.g. Sugitani et al. 1995; White et al. 1997; Lefloch et al. 1997, and references therein). However, the interest about IC $1396 \mathrm{~N}$ has very much increased recently due to observations at far-infrared (FIR) wavelengths with the Infrared Space Observatory (ISO). Such observations have unvealed a rich spectrum, with many lines of $\mathrm{CO}, \mathrm{OH}$ and $\mathrm{H}_{2} \mathrm{O}$, indicating the presence within the globule of 
dense shocked regions excited at a temperature in excess of $1500 \mathrm{~K}$ (Saraceno et al. 1996, 2001). It is worth noting that, compared to e.g. Orion A, which is illuminated by clusters of O-B stars and associated with more massive molecular clouds, the structure of the IC 1396 region is less complex. Thus, IC $1396 \mathrm{~N}$ become one of the best laboratories in which investigate the mechanisms of star formation induced by the propagation of an ionization shock front, the so-called radiation driven implosion (RDI) mechanism (Reipurth 1983).

We have investigated the bright rimmed globule IC $1396 \mathrm{~N}$ through a multiline survey at mm-wavelengths which allow to probe a wide range of physical conditions. Moreover, in order to study the IC $1396 \mathrm{~N}$ young stellar content, we have carried out interferometric continuum observations at three different frequencies (88, 92, and $110 \mathrm{GHz}$ ). The main aims of this work are: (i) to carefully investigate the structure of the globule and of the bipolar outflow with high spatial and spectral resolution (through $\mathrm{CO}$ and $\mathrm{HCO}^{+}$observations), (ii) to trace the high density structures at velocities close to the ambient one (through $\mathrm{CS}$ and $\mathrm{DCO}^{+}$) and the highest excitation conditions of the molecular outflow (through $\mathrm{SiO}$ ), (iii) to study how the presence of massive stars can affect the structure of the dense molecular clouds in the surroundings triggering, at the same time, the process of star formation. In addition, the present survey allows to compare high resolution data with theoretical models and, in particular with the numerical simulations of a photo-ionized globule presented by Lefloch \& Lazareff $(1994,1995)$.

\section{Observations}

\subsection{Single-dish observations}

Observations with the IRAM 30-m telescope at Pico Veleta (near Granada, Spain) were carried out during several runs in November 1998 and January and May 1999. Table 1 summarizes the observed molecular species, the transitions, their rest frequencies and some observing parameters, such as the $H P B W$, the typical system temperature $\left(T_{\text {sys }}\right)$, the integration time $\left(t_{\text {int }} ; \mathrm{ON}+\mathrm{OFF}\right.$ source $)$ and the spectral resolution $(\mathrm{d} v)$. The main beam efficiency varies from 0.75 (at $90 \mathrm{GHz}$ ) to 0.37 (at $230 \mathrm{GHz}$ ) according with the values reported by Wild (1995). The observations were made by position switching. Pointing was checked every hour by observing nearby planets or continuum sources and it was found to be accurate to within $3^{\prime \prime}$. As spectrometers, an autocorrelator (AK) split into three parts was used to allow simultaneous observations of three different transitions. Moreover, also a $1 \mathrm{MHz}$ filter bank, split into three parts of $2 \times 256$ and 512 channels, was used to allow simultaneous observations. The velocity resolutions provided by both backends, AK and $1 \mathrm{MHz}$, are shown in Table 1 . The spectra were calibrated with the standard chopper wheel method and are reported here in units of main-beam brightness temperature $\left(T_{\mathrm{MB}}\right)$.

\subsection{Interferometric observations}

The observations with the Owens Valley Radio Observatory (OVRO) mm-array located near Big Pine (CA, USA) have been performed during June 1997. The six 10.4-m dishes were employed in the low resolution configuration providing baselines between 15 and $115 \mathrm{~m}$. IC $1396 \mathrm{~N}$ was observed in the continuum at 88, 92, and $110 \mathrm{GHz}$ and in the $\mathrm{HCO}^{+} J=1-0$ line at $89188.523 \mathrm{MHz}$. The phase center corresponds to the coordinates of the strongest $\mathrm{H}_{2} \mathrm{O}$ maser source as detected by Tofani et al. (1995), which are slightly offset $\left(-4^{\prime \prime},+2^{\prime \prime}\right)$ with respect to the IRAS position (see Sect. 3). For the line observations we configured the flexible digital correlator to observe the line within two bands simultaneously with spectral resolutions of 1.7 and $0.4 \mathrm{~km} \mathrm{~s}^{-1}$ and bandwidths of 105 and $25 \mathrm{~km} \mathrm{~s}^{-1}$, respectively. The continuum observations used an analog correlator with $1 \mathrm{GHz}$ wide bands (placed in the upper and/or lower sidebands). Frequent observations of BL Lac (2.3-3.0 Jy), with an integration time of $8 \mathrm{~min}$ each $\sim 20 \mathrm{~min}$, were used to perform phase and gain calibration. The passband calibration was carried out observing either 3C 273 (25-33 Jy) or 3C 454.3 (4.3$5.3 \mathrm{Jy}$ ) and BL Lac. The flux density scale was derived by observations of Neptune and Uranus, the calibration uncertainty is expected to be $\simeq 20 \%$. All the calibration has been performed using the MMA software (Scoville et al. 1993). The calibrated data have been loaded into the NRAO AIPS package and imaged and cleaned using the IMAGR task. Continuum maps and line data cubes were produced using both natural and robust weighting of the $(u, v)$ data. The typical synthesised beams and noise level achieved are reported in Table 2. Continuum subtraction have been performed on the image cubes by using the continuum maps, due to the wide band used in the continuum observations this method yields a much better signal-to-noise than using the line free channels of the line observations.

\section{The globule and the outflows}

Figure 1 shows the maps of the integrated CO $J=$ $2-1$ and $\operatorname{CS} J=3-2$ emissions, centred at $\alpha_{2000}=$ $21^{\mathrm{h}} 40^{\mathrm{m}} 42^{\mathrm{s}} \cdot 3, \delta_{2000}=+58^{\circ} 16^{\prime} 10^{\prime \prime}$, i.e. the coordinates of IRAS $21391+5802$. These new maps, which are in agreement with previous lower resolution $\mathrm{CO}$ data (e.g. Weikard et al. 1996) and less complete CS data (Serabyn et al. 1993), reveal the structure of the globule with unprecedented detail. The $\mathrm{CO}$ map brings to light the cometary nature of the globule, with a remarkable headtail structure. The CS observations show that the high density region of IC $1396 \mathrm{~N}$ is about $120^{\prime \prime}(\simeq 0.4 \mathrm{pc})$ wide and $\simeq 220^{\prime \prime}(0.8 \mathrm{pc})$ long. The axis of the elongated structure points towards HD 206267, whose direction is indicated in Fig. 1 by the arrows.

Figure 2 reports examples of molecular line profiles observed towards different positions in the IC $1396 \mathrm{~N}$ region. 
Table 1. List of molecular species, transitions and observing parameters (IRAM 30-m antenna).

\begin{tabular}{l|l|c|c|c|c|c|c}
\hline Molecules & Transition & $\begin{array}{c}\text { Rest frequency } \\
(\mathrm{MHz})\end{array}$ & $\begin{array}{c}H P B W \\
\left({ }^{\prime \prime}\right)\end{array}$ & $\begin{array}{c}T_{\text {sys }} \\
(\mathrm{K})\end{array}$ & $\begin{array}{c}t_{\text {int }} \\
(\mathrm{s})\end{array}$ & $\begin{array}{c}\mathrm{d} v(\mathrm{AK}) \\
\left(\mathrm{km} \mathrm{s}^{-1}\right)\end{array}$ & $\begin{array}{c}\mathrm{d} v(1 \mathrm{MHz}) \\
\left(\mathrm{km} \mathrm{s}^{-1}\right)\end{array}$ \\
\hline $\mathrm{SiO}$ & $J=2-1$ & 86846.998 & 29 & 150 & 320 & 0.13 & 3.45 \\
$\mathrm{CO}$ & $J=1-0$ & 115271.195 & 21 & 280 & 75 & 0.10 & 2.60 \\
$\mathrm{SiO}$ & $J=3-2$ & 130268.702 & 18 & 220 & 320 & 0.17 & 2.30 \\
$\mathrm{DCO}^{+}$ & $J=2-1$ & 144077.328 & 16 & 370 & 150 & 0.21 & - \\
$\mathrm{CS}$ & $J=3-2$ & 146969.047 & 16 & 280 & 75 & 0.32 & 2.04 \\
$\mathrm{SiO}$ & $J=5-4$ & 217104.935 & 11 & 270 & 320 & 0.11 & 1.38 \\
$\mathrm{CO}$ & $J=2-1$ & 230537.984 & 10 & 290 & 75 & 0.10 & 1.30 \\
\hline
\end{tabular}

Table 2. Instrumental parameters of the OVRO array observations.

\begin{tabular}{l|c|c|c}
\hline \multicolumn{1}{c|}{ Parameters } & $86-89 \mathrm{GHz}$ & $92 \mathrm{GHz}$ & $110 \mathrm{GHz}$ \\
\hline Sideband tuning & Double & Single (Lower) & Single (Lower) \\
Local Osc. Freq. (GHz) & 88.01776 & 93.72964 & 111.59109 \\
Primary beam $F W H P$ & $72^{\prime \prime}$ & $66^{\prime \prime}$ & $54^{\prime \prime}$ \\
Synthesised $H P B W$ & Natural weight.: $6 . .7 \times 4^{\prime \prime} 8\left(-66^{\circ}\right)$ & & \\
& Robust weight.: $6^{\prime \prime} .2 \times 3^{\prime \prime} .4\left(-68^{\circ}\right)$ & $5^{\prime \prime} 4 \times 4^{\prime \prime} 0\left(-56^{\circ}\right)$ & $5^{\prime \prime} .2 \times 3^{\prime \prime} 0\left(-50^{\circ}\right)$ \\
\hline
\end{tabular}

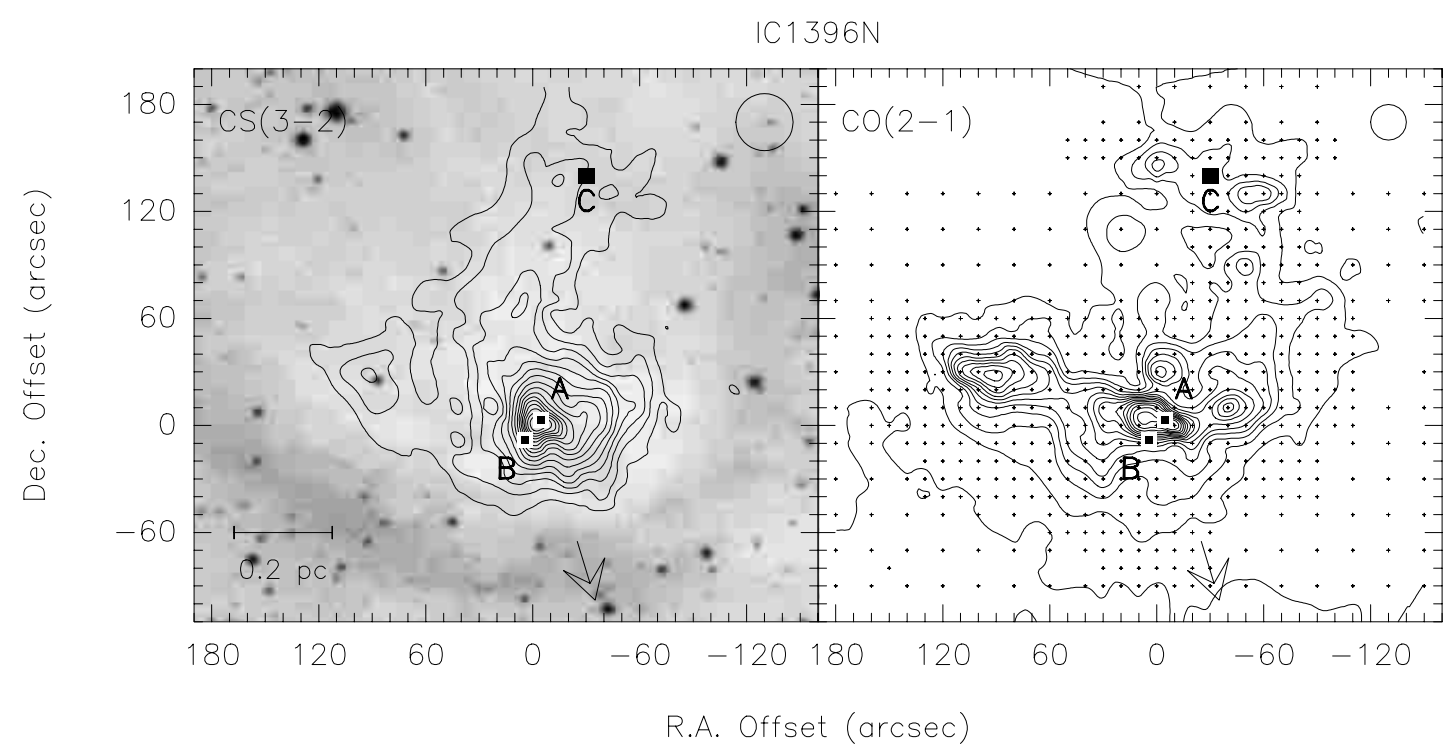

Fig. 1. Contour maps of the integrated CO $(J=2-1)$ and CS $(J=3-2)$ emission towards IC $1396 \mathrm{~N}$ with superimposed the optical image reproduced from the POSS red plates. The velocity integration intervals are $-60,+60 \mathrm{~km} \mathrm{~s}^{-1}$ for $\mathrm{CO}$ and -9 , $+9 \mathrm{~km} \mathrm{~s}^{-1}$ for CS. The empty circles show the IRAM beam $(H P B W)$, the small crosses mark the observed positions, while the arrows point the direction where, at a projected distance of $11 \mathrm{pc}$, HD 206267 is located. The three squares point out the three continuum sources detected using the OVRO interferometer (A and B, Sect. 4.2.1) and the IRAM antenna (C, Sect. 3.1). The CO contours range from $10.0\left(\sim 10 \sigma\right.$, where $\sigma$ is the rms of the map) to $465.0 \mathrm{~K} \mathrm{~km} \mathrm{~s}^{-1}$ by step of $30.0 \mathrm{~K} \mathrm{~km} \mathrm{~s}$. The CS contours range from $2.5(5 \sigma)$ to $45.0 \mathrm{~K} \mathrm{~km} \mathrm{~s}^{-1}$ by step of $2.5 \mathrm{~K} \mathrm{~km} \mathrm{~s}^{-1}$.

The CO spectra clearly show high velocity wings, confirming the occurrence of molecular outflows in the IC $1396 \mathrm{~N}$ interior: the emission velocity interval extends up to -35 and about $+55 \mathrm{~km} \mathrm{~s}^{-1}$, in agreement with the CO $J=$ 3-2 results of Serabyn et al. (1993) and extending the velocity range detected by Sugitani et al. (1989). Figure 3 reports the channel map of the CO $J=2-1$ emission, allowing to study the gas kinematics. The ambient LSR velocity emission (see Sect. 4.1) is centred around the -1.5 and $+1.5 \mathrm{~km} \mathrm{~s}^{-1}$ panels (underlined by thicker boxes): the strong self-absorption in the CO spectra (see Fig. 2) does not allow one to define the cloud structure at these velocities. From Fig. 3, it is possible to see that: (i) high velocity blue- and redshifted emission is coming from a bipolar jetlike structure symmetrically located with respect to the IRAS $21391+5802$ coordinates, (ii) there is a significant 
spatial overlap between the blue and the red emissions, (iii) other two high velocity elongated components located along a direction similar to that of the structure individuated around the IRAS counterpart are detected at about Dec. Offset $=+140^{\prime \prime}$. We conclude that IC $1396 \mathrm{~N}$ is associated with at least two molecular outflows: that located around the $\left(0^{\prime \prime}, 0^{\prime \prime}\right)$ position, hereafter called central outflow, and that located around the $\left(-30^{\prime \prime},+140^{\prime \prime}\right)$ offset, hereafter called northern outflow. The length of the central and the northern $\mathrm{CO}$ outflows is about 0.7 and $0.3 \mathrm{pc}$, while the collimation factor (ratio between length and width) is $\sim 4.5$ and 3 , respectively.

In the region of the globule between the central and the northern outflows, roughly around the $\left(-30^{\prime \prime},+90^{\prime \prime}\right)$ position, red- and blueshifted extended structures are clearly detected at velocities relatively close $\left(\leq \pm 10 \mathrm{~km} \mathrm{~s}^{-1}\right)$ to the ambient one. In addition, a red clump at $\left(0^{\prime \prime},+30^{\prime \prime}\right)$ and a blue one at $\left(-40^{\prime \prime},+20^{\prime \prime}\right)$ are observed. This emission could indicate the occurrence of other outflows, as supported also by the detection at near IR wavelengths of faint $\mathrm{H}_{2}$ knots (Nisini et al. 2001). However, contribution to the low velocity red- and blueshifted emission can also come from gas that could be photo-evaporating from the external surface of the globule. This possibility, which is discussed in Sect. 5, is supported by the low velocity panels (both red- and blueshifted) of Fig. 3 which clearly trace the rim-shape of IC $1396 \mathrm{~N}$, as expected if a significant amount of gas were leaving the globule surface at moderate velocities.

\subsection{The northern outflow}

Although the central outflow was previously known, the present data provide the first clear evidence for the existence of a bipolar molecular outflow in the northern region of IC $1396 \mathrm{~N}$. It is worth noting that recent $2.12 \mu \mathrm{m}$ IR observations of IC 1396N (Nisini et al. 2001, see their Fig. 4) reveal several $\mathrm{H}_{2}$ emission knots in this region of the globule; in particular, two chains of $\mathrm{H}_{2}$ knots are coincident with the two lobes of the northern outflow. The channel maps of Fig. 3 provide some information on the structure of the northern outflow. It shows a blueshifted lobe, NW, at $\left(-60^{\prime \prime},+130^{\prime \prime}\right)$ which emits up to $\simeq-25 \mathrm{~km} \mathrm{~s}^{-1}$, and a redshifted component, $\mathrm{NE}$, at $\left(0^{\prime \prime},+150^{\prime \prime}\right)$ with velocities up to about $+15 \mathrm{~km} \mathrm{~s}^{-1}$. Taking into account the definite spatial separation between blue and red emissions, we estimate that the inclination angle of the outflow to the plane of the sky is about $10^{\circ}$ (see Table 3 ).

In order to search the driving source of this outflow, we performed continuum mm-wave observations with the IRAM 30-m radiotelescope equipped with the 19 channel MPIfR bolometer (for more details about the $30-\mathrm{m}$ continuum observations see e.g. Tafalla et al. 1999). We explored a region of about $100^{\prime \prime} \times 100^{\prime \prime}$ in the northern part of IC $1396 \mathrm{~N}$. An unresolved source of $40 \mathrm{mJy}$ was detected with a $S / N=4$ at the $\left(-30^{\prime \prime},+140^{\prime \prime}\right)$ offset, i.e. exactly at the coordinates predicted from the $\mathrm{CO} J=2-1$ map of the outflow. This source is designated in the maps as IC 1396 N-C. Since the C continuum source has been observed only at $1.25 \mathrm{~mm}$, we cannot derive an the integrated luminosity from its spectral energy distribution. However, following the procedure described in Sect. 4.2.1, the mass of the dust condensation can be derived: $2 M_{\odot}$. Using this value, a rough estimation of the bolometric luminosity, $\sim 10 L_{\odot}$, can be then obtained by means of the relationships given e.g. by Bontemps et al. (1996) and Bachiller \& Tafalla (1999).

\subsection{Structure of the central outflow}

The main bipolar outflow in IC $1396 \mathrm{~N}$ was previously studied by Sugitani (1989). However, the data reported here provide new interesting information on its structure. The CO channel map reveals different clumps along the outflow axis. The eastern lobe clearly shows two knots, one at $\left(+90^{\prime \prime},+30^{\prime \prime}\right)$, designated as SE1, detected up to $\simeq-40 \mathrm{~km} \mathrm{~s}^{-1}$, and one at $\left(+10^{\prime \prime}, 0^{\prime \prime}\right)$, SE2, which emits at lower velocities, up to about $-26 \mathrm{~km} \mathrm{~s}^{-1}$. Also the western lobe has two knots, one at $\left(-40^{\prime \prime},+10^{\prime \prime}\right)$, SW1, detectable up to about $+20 \mathrm{~km} \mathrm{~s}^{-1}$, and one at $\left(0^{\prime \prime}, 0^{\prime \prime}\right)$, $\mathrm{SW} 2$, up to $\simeq+40 \mathrm{~km} \mathrm{~s}^{-1}$. In summary, we detect four clumps (pointed out in the channel maps) in the central outflow: SE1 $\left(+90^{\prime \prime},+30^{\prime \prime}\right)$, SE2 $\left(+10^{\prime \prime}, 0^{\prime \prime}\right)$, SW2 $\left(0^{\prime \prime}, 0^{\prime \prime}\right)$ and SW1 $\left(-40^{\prime \prime},+10^{\prime \prime}\right)$. This clumpy structure suggests that episodic events of the mass loss process have occurred. The outflow morphology suggests that the driving source has to be located very close to the $\left(0^{\prime \prime}, 0^{\prime \prime}\right)$ offset, i.e. around the IRAS coordinates (see Sect. 4.2).

The jet-like structure of the outflow is clearly underlined at velocities higher than about $\pm 10 \mathrm{~km} \mathrm{~s}^{-1}$ (see Fig. 3). However, the panels corresponding to low velocities $\left( \pm 3 \mathrm{~km} \mathrm{~s}^{-1}\right)$ with respect to the ambient one already allow us to identify the main structures of the outflow, suggesting that a considerable fraction of the outflow mass is due to low velocity swept-up material. It is worth noting that while the eastern lobe is $130^{\prime \prime}$ length and is located along the northeast-southwest direction, the western counterpart is less extended and bends towards north at the lobe end. This configuration can be explained by the interaction of the outflow with the ambient medium, which, as the mass lost travels towards the high density western part of the globule (see the CS map in Fig. 1), slows down and changes the direction of motion of the flowing gas.

\subsection{Physical parameters obtained from the CO data}

In order to estimate opacities and excitation temperatures $\left(T_{\mathrm{ex}}\right)$, the spectra of the two $\mathrm{CO}$ transitions have been compared, after convolving the $J=2-1$ spectra to the spatial resolution of the $J=1-0$ observations $\left(H P B W=21^{\prime \prime}\right)$. Several positions corresponding to the different lobes of the outflows have been considered. The convolved spectra show that in general the peak mainbeam brightness temperature is lower, indicating that the 


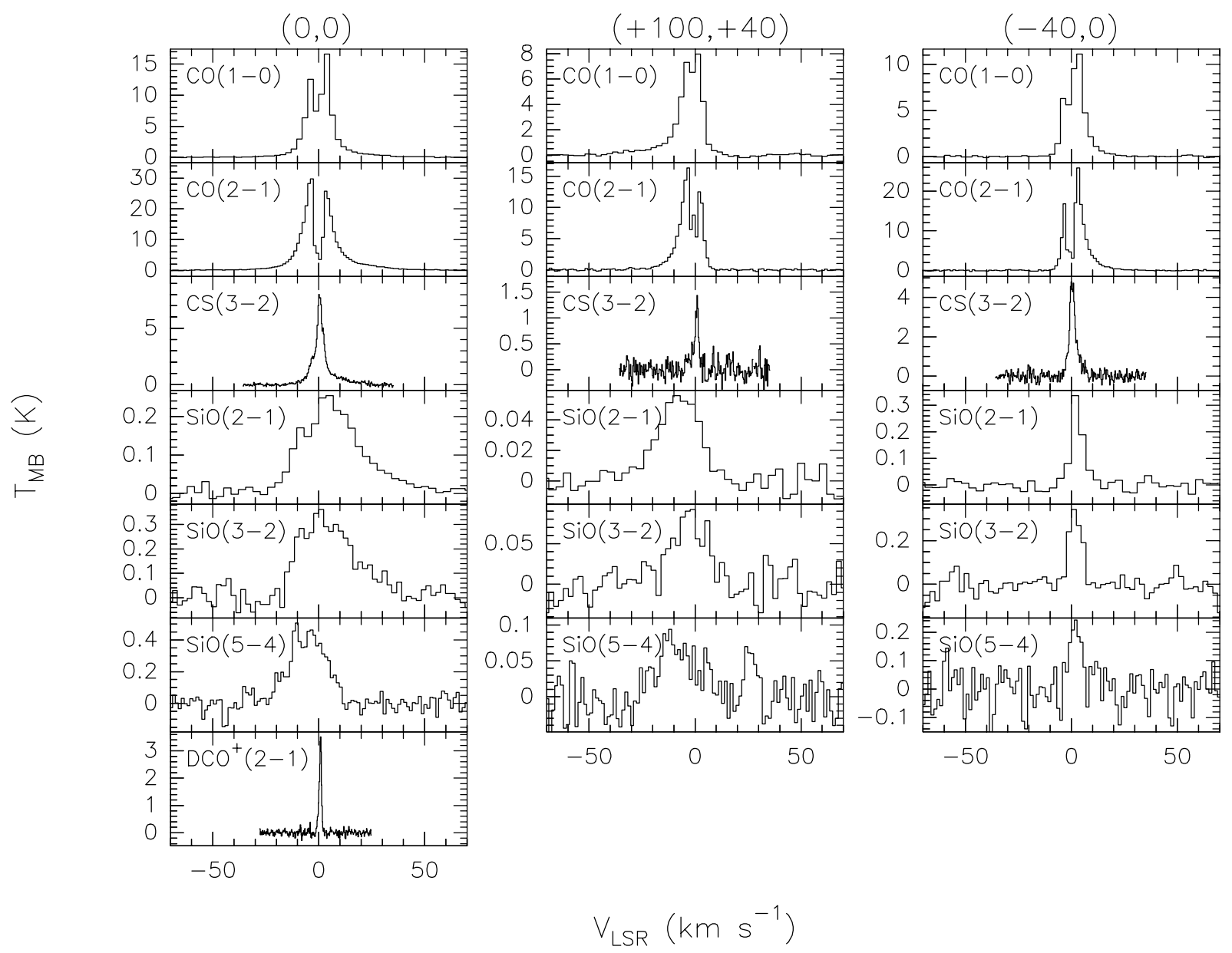

Fig. 2. Examples of molecular line profiles observed towards three positions in the IC $1396 \mathrm{~N}$ central outflow (see text) with the 30-m IRAM antenna. Molecular species, transition and angular offset are indicated.

bulk of the high velocity emission is due to compact structures, smaller than the beam (see Fig. 3). An exception comes from the western lobes of the two outflows, where the peak $T_{\mathrm{MB}}$ roughly holds the same values: this could be due to the more extended $\mathrm{CO}$ structures detected in Fig. 3 in the western part of IC $1396 \mathrm{~N}$ at velocities $\leq 10 \mathrm{~km} \mathrm{~s}^{-1}$ which fill the $J=1-0$ beam. Moreover, the temperature of the self-absorption feature (see Fig. 2) remains the same after the convolution, indicating that the cool gas $(\leq 10 \mathrm{~K})$ responsible of the absorption is located in a quite extended region.

For the central outflow, the ratio $R$ between the observed brightness temperatures of the CO $J=2-1$ and $J=1-0$ transitions is about 0.9 for the optically thick emission at low velocities, suggesting (see Fig. 12 of Levreault 1988) an excitation temperature around $20 \mathrm{~K}$. Given the uncertainties in the data calibration and the dependence of $R$ on $T_{\text {ex }}$ in the thick case, the value $R=0.9$ does not allow to derive a reliable excitation temperature. However, taking into account also the $T_{\mathrm{MB}}$ values shown by the CO spectra of Fig. 2, it is reasonable to assume $T_{\text {ex }}=20 \mathrm{~K}$. Moving towards higher velocities along the CO spectra, $R$ monotonically reaches higher values, up to 2.2. This trend has already been found for well-known molecular outflows and can be due either to a variation of the excitation temperature or, more probably, to the decreasing opacity at higher velocities, where a smaller amount of flowing gas is usually observed. The highest $R$ values for the central outflow can be explained by a $T_{\text {ex }}$ of $15-20 \mathrm{~K}$ and a $J=2-1$ optical depth $\tau \leq 0.2$. For the northern outflow, we have $R \simeq 1$ for the low velocity emission, which can be obtained with an excitation temperature of about $10-20 \mathrm{~K}$ and an optical depth of $\sim 2-6$. At the highest velocities $R \simeq 2$ : if we assume $T_{\mathrm{ex}}=20 \mathrm{~K}$, we infer $\tau \sim 0.4$, whereas $T_{\text {ex }}=15 \mathrm{~K}$ leads to lower optical depths.

The kinematical parameters of the two detected outflows in IC $1396 \mathrm{~N}$, reported in Table 3 , have been calculated following the standard procedure reported e.g. by Lada (1985) and assuming an excitation temperature of $20 \mathrm{~K}$ and $10 \mathrm{~K}$ for the central and the northern outflow, respectively. A relative $\mathrm{CO}$ abundance of $10^{-4}$ has been used. Following the above reported results, we have assumed for both outflows thick emission for the intermediate velocities $(\tau=10$ and $=2$ for the central and northern outflows, respectively) and thin emission ( $\tau$ equals to 0.02 and 0.2 ) for the high velocities (above $20 \mathrm{~km} \mathrm{~s}^{-1}$ with respect to the ambient one). 


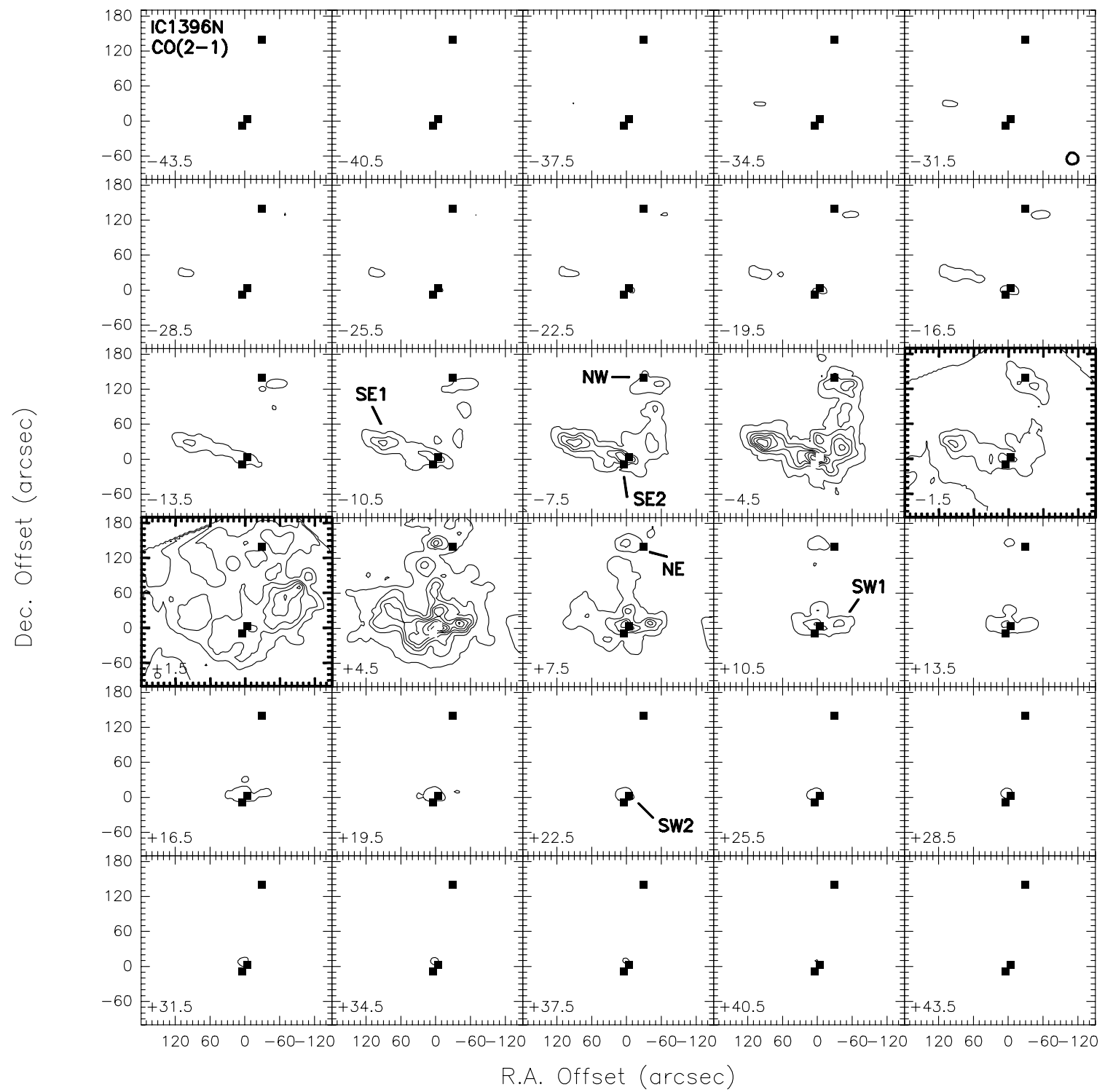

Fig. 3. Channel map of the CO $J=2-1$ emission towards IC $1396 \mathrm{~N}$. Each panel shows the emission integrated over a velocity interval of $3 \mathrm{~km} \mathrm{~s}^{-1}$ centred at the value given in the left corner. The thick boxes point out the ambient velocity emission (see Sect. 4.1). Symbols are drawn as in Fig. 1, while the labels underline different components of the molecular outflow (see Sect. 3). The contours range from $3.00(\sim 5 \sigma)$ to $87.00 \mathrm{~K} \mathrm{~km} \mathrm{~s}^{-1}$ by step of $12.00 \mathrm{~K} \mathrm{~km} \mathrm{~s}^{-1}$.

Table 3. Dynamical parameters of the IC $1396 \mathrm{~N}$ outflows.

\begin{tabular}{|c|c|c|c|c|c|c|c|c|}
\hline & $\begin{array}{c}\theta \\
\left({ }^{\circ}\right)\end{array}$ & $\begin{array}{c}M \\
\left(M_{\odot}\right)\end{array}$ & $\begin{array}{c}P \\
\left(M_{\odot} \mathrm{km} \mathrm{s}^{-1}\right)\end{array}$ & $\begin{array}{c}E_{\text {kin }} \\
\left(10^{44} \mathrm{erg}\right)\end{array}$ & $\begin{array}{c}t \\
\left(10^{4} \mathrm{yr}\right)\end{array}$ & $\begin{array}{c}F \\
\left(M_{\odot} \mathrm{km} \mathrm{s}^{-1} \mathrm{yr}^{-1}\right)\end{array}$ & $\begin{array}{c}\dot{M} \\
\left(M_{\odot} \mathrm{yr}^{-1}\right)\end{array}$ & $\begin{array}{c}L_{\mathrm{m}} \\
\left(L_{\odot}\right)\end{array}$ \\
\hline \multirow[t]{2}{*}{ Northern O. } & - & 0.01 & 0.1 & 0.1 & 9.4 & $9.2 \times 10^{-7}$ & $1.1 \times 10^{-7}$ & $1 \times 10^{-3}$ \\
\hline & 10 & 0.01 & 0.4 & 2.4 & 1.7 & $3.0 \times 10^{-5}$ & $6.2 \times 10^{-7}$ & 0.19 \\
\hline \multirow[t]{3}{*}{ Central O. } & - & 0.10 & 0.7 & 0.6 & 8.5 & $8.6 \times 10^{-6}$ & $1.2 \times 10^{-6}$ & $6 \times 10^{-3}$ \\
\hline & 10 & 0.10 & 3.8 & 19.5 & 1.5 & $2.8 \times 10^{-4}$ & $6.6 \times 10^{-6}$ & 1.17 \\
\hline & 20 & 0.10 & 1.9 & 5.0 & 3.1 & $6.9 \times 10^{-5}$ & $3.2 \times 10^{-6}$ & 0.15 \\
\hline
\end{tabular}

The central outflow has a total mass of $0.1 M_{\odot}$ equally distributed between the red and the blue lobes. In order to derive the kinematical outflow parameters, the angle $\theta$ of inclination to the plane of the sky has to be assumed. Unfortunately, because of the complexity of the region, the present observations do not allow a good $\theta$ estimation. However, taking a compromise between the quite elongated structure of the outflow and the overlap between the two lobes (see the velocity channel maps in Fig. 3), it is reasonable to assume a low $\theta$ value, 
probably in the $10^{\circ}-20^{\circ}$ range. For orientation, we give in Table 3 the kinematical parameters for such $\theta$ values together with those calculated without any correction due to geometry assumptions. Thus, the estimate of the momentum and the kinetic energy gives $P=1.9-3.8 M_{\odot} \mathrm{km} \mathrm{s}^{-1}$ and $E_{\text {kin }}=(5-20) \times 10^{44} \mathrm{erg}$, respectively. A kinematical timescale of $\simeq 2-3 \times 10^{4}$ yr has been derived, allowing one to deduce the force required to drive the mass loss process, $F=6.9 \times 10^{-5}-2.8 \times 10^{-4} M_{\odot} \mathrm{km} \mathrm{s}^{-1} \mathrm{yr}^{-1}$, the mass loss rate, $\dot{M}=(3.2-6.6) \times 10^{-6} M_{\odot} \mathrm{yr}^{-1}$, and the mechanical power, $L_{\mathrm{m}}=0.15-1.17 L_{\odot}$. It is worth noting that the uncorrected parameters listed in Table 3 slightly differentiate from those calculated without any geometry correction by Sugitani et al. (1989), presenting a more energetic outflow. This discrepancy can be understood by taking into account that the present observations have detected CO emission at higher velocities, up to about $50 \mathrm{~km} \mathrm{~s}^{-1}$ with respect to the ambient one. Given the expected correlation between the outflow dynamical parameters and the source bolometric luminosity (e.g. Cabrit \& Bertout 1992 and references therein) we infer for the driving source $L_{\text {bol }} \sim 3 L_{\odot}$, in case of $\theta=20^{\circ}, \sim 100 L_{\odot}$, if $\theta=10^{\circ}$. These values will be compared in Sect. 4.2.1 with other $L_{\text {bol }}$ estimates derived through observations at different wavelengths. Finally, it is worth noting that the derived mechanical power, $0.15-1.17 L_{\odot}$, is in agreement with that calculated using emission detected by ISO and due to high excitation CO transitions by Nisini et al. (1998). The authors obtained $0.63 L_{\odot}$ assuming a definite correlation between $L_{\mathrm{m}}$ and the luminosity radiated away by the shock traced by ISO CO lines.

With a mass of only $\sim 0.01 M_{\odot}$, the northern outflow is found to be an order of magnitude less massive than the central one. The outflow momentum is $P=0.4 M_{\odot} \mathrm{km} \mathrm{s}^{-1}$, its kinetic energy $2.4 \times 10^{44} \mathrm{erg}$, while the estimate of the kinematical timescale leads to $\sim 1.7 \times 10^{4} \mathrm{yr}$. The force of the northern outflow is $3 \times 10^{-5} M_{\odot} \mathrm{km} \mathrm{s}^{-1} \mathrm{yr}^{-1}$, its mass loss $\simeq 6 \times 10^{-7} M_{\odot} \mathrm{yr}^{-1}$ and its mechanical power $0.19 L_{\odot}$. These parameters suggest that the bolometric luminosity of the driving source is $\sim 4 L_{\odot}$, a value in reasonable agreement with the estimation obtained using the mass of the dust condensation associated with the $\mathrm{C}$ continuum source $\left(\sim 10 L_{\odot}\right)$.

Figure 4 shows the distribution of the outflow wing intensity with velocity (not corrected for the inclination angles) for both outflows. If the emission is optically thin, the brightness distribution is proportional to that of the flow mass. In the case of a constant optical depth along the wings, thicker emission would affect the distribution only moving down the points in the vertical axis by a same quantity, thus leaving unchanged the plot shape. Recently, it has pointed out that the outflow brightness distributions can be described by a power law $T_{\mathrm{MB}} \propto\left|v-v_{0}\right|^{\gamma}$ : the slope is about -1.5 for low velocities, while at higher speeds it becomes steeper, reaching values of the order of -4 (Lada \& Fich 1996; Bachiller \& Tafalla 1999 and references therein). The occurrence of a break-point could be due to high velocity gas which slows down because

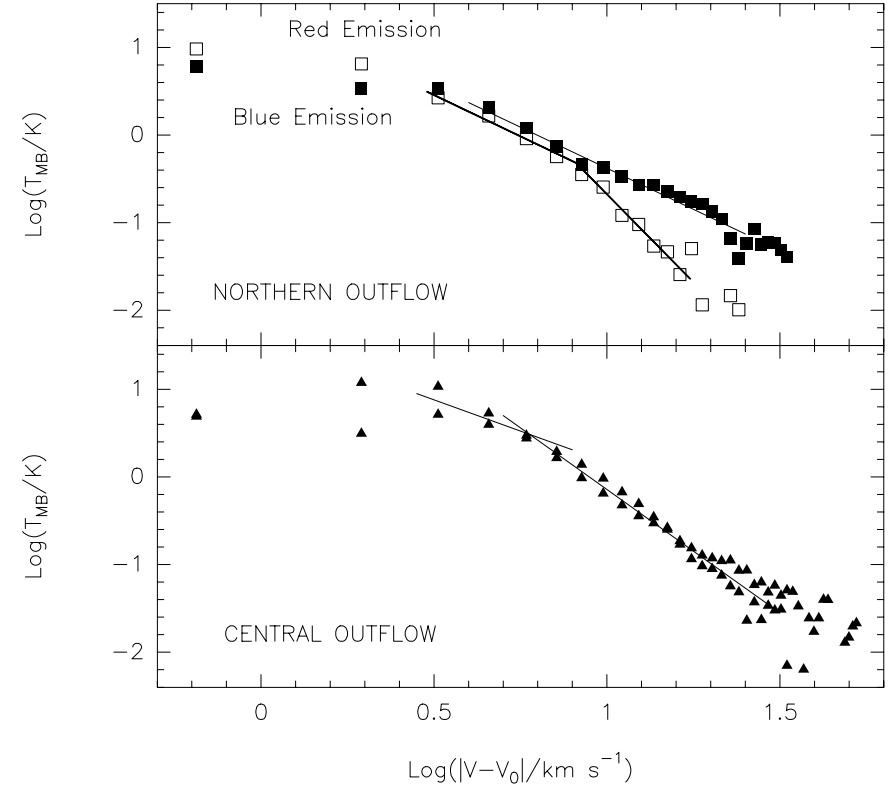

Fig. 4. Brightness distribution with velocity from the central source $\left(\left|v-v_{0}\right|\right)$ of the IC $1396 \mathrm{~N}$ northern (upper panel) and central (lower panel) outflow. Continuous lines stand for the separate fits $T_{\mathrm{MB}} \propto\left|v-v_{0}\right|^{\gamma}$. For the northern outflow two plots have been drawn: filled squares (thin line) for the blue lobe and empty squares (thick line) for the red one.

the interaction with the surrounding medium, producing a deflection in the power-law slope. It is reasonable to assume that the younger outflows are associated with faster gas with respect to the more evolved ones. Therefore, the relative fraction of slow material with respect to the fast gas should increase with time, shifting the position of the break-point in the distribution.

Figure 4 shows that also the IC $1396 \mathrm{~N}$ brightness distributions can be described by a power-law. For the central outflow we have $\gamma=-1.4$ at low velocities $\left(\leq 6-8 \mathrm{~km} \mathrm{~s}^{-1}\right)$, while at higher velocities $\gamma$ is about -2.8 . This distribution is not easy to interpret since the break-point at low velocities resembles those of quite evolved outflows (see Fig. 10 of Bachiller \& Tafalla 1999), whereas at the same time it presents a remarkable emission at quite high velocities. The possible presence of other outflows along the line of sight of the central one (including perhaps photoevaporating gas from the globule surface) could provide an explanation for the plot shown in the lower panel of Fig. 4. On the other hand, the two lobes of the northern outflow have different plots, which have been drawn through filled (blue lobe) and empty (red lobe) squares. Blue emission is represented by a power-law with $\gamma=-1.9$, while red emission can be fitted with -1.9 till about $10 \mathrm{~km} \mathrm{~s}^{-1}$ and then the distribution becomes steeper with $\gamma=-3.3$. These brightness distributions seem to indicate that the northern outflow is younger than the central one. The difference between the break point velocities of the northern (6-8 $\left.\mathrm{km} \mathrm{s}^{-1}\right)$ and the central $\left(10 \mathrm{~km} \mathrm{~s}^{-1}\right)$ outflows could be smoothed away if the central outflow was closer to the plane of the sky with respect to the northern one by a 
factor of 1.4, but this seems in contrast with the scenario drawn above following the CO channel maps. The difference between the plots of the blue- and redshifted emission could reflect a difference of the physical conditions of the surrounding medium found by the two bipolar outflow motions. If the ambient medium met by the gas associated with the eastern lobe was denser than that of the gas to the west direction, then the red lobe could be more efficiently slowed down, leading to the observed brightness distributions. This scenario is supported by the present CS observations: while towards the position of the western blue lobe a relatively weak $\left(\simeq 2.3 \mathrm{~K} \mathrm{~km} \mathrm{~s}^{-1}\right)$ narrow $\left(\simeq 1 \mathrm{~km} \mathrm{~s}^{-1}\right)$ emission has been observed, in correspondence of the eastern red lobe CS shows a broad emission spread over a velocity range of about $6 \mathrm{~km} \mathrm{~s}^{-1}$, suggesting a close interaction of the outflow with the ambient medium.

\subsection{SiO emission from the central outflow}

In order to clarify the structure of the high velocity gas components of the IC $1396 \mathrm{~N}$ central outflow, a standard tracer such as $\mathrm{SiO}$ has been used. Figure 5 reports the contour maps of the integrated $\mathrm{SiO} J=2-1, J=3-2$ and $J=5-4$ emission. Silicon monoxide is present only along the main axis detected through carbon monoxide, confirming its well-known enhancement in molecular outflows (e.g. Martín-Pintado et al. 1992; Codella et al. 1999). In particular, Fig. 5 shows the occurrence of a $\mathrm{SiO}$ structure which is located slightly to the west with respect to the central position (one step of the map sampling) and which grows its importance with excitation.

In order to investigate the outflow kinematics, the velocity channel maps of the $J=5-4$ emission, e.g. that observed with the best spectral and spatial resolution, have been reported in Fig. 6. It is possible to clearly observe the elongated structure of the outflow, with the four clumps detected in CO. While the SE1 clump is spatially unresolved $(\leq 0.04 \mathrm{pc})$, the size of the other clumps is about $0.1 \mathrm{pc}$, while the collimation factor is about 6 , i.e. a value somewhat larger than that derived using CO $(\sim 4-5)$, confirming that $\mathrm{SiO}$, with respect carbon monoxide, can trace the inner part of the outflow.

The $\mathrm{SiO}$ emission presents different linewidths in different map positions (see Fig. 2): broad emission ( $F W H M \simeq 25-30 \mathrm{~km} \mathrm{~s}^{-1}$ for the $J=2-1$ transition) has been detected towards the SE1, SE2 and SW2 clumps, whereas at the $\left(-40^{\prime \prime}, 0^{\prime \prime}\right)$ position, where the SW1 clump is located, the linewidth is quite narrow $\left(\simeq 5 \mathrm{~km} \mathrm{~s}^{-1}\right)$. The narrow $\mathrm{SiO}$ emission can be explained, following Lefloch et al. (1998) and Codella et al. (1999), as a signature of the evolution of the shocked gas: the $\mathrm{SiO}$ component, produced in high velocity shock-compressed regions, is slowed down because of the interaction with the ambient gas hosting the star forming process. Therefore, in the specific case of the IC $1396 \mathrm{~N}$ central outflow, the narrow $\mathrm{SiO}$ emission at the ambient LSR velocity towards the SW1 clump can be interpreted as a result of the interaction between the outflow motion and the high density medium located in the western direction.

Moreover, the $\mathrm{SiO}$ spectra of the IC $1396 \mathrm{~N}$ central outflow reveal the signature of intense emission at about $-10 \mathrm{~km} \mathrm{~s}^{-1}$ towards the central positions. As an example, the $\mathrm{SiO}$ line profiles of the $\left(0^{\prime \prime}, 0^{\prime \prime}\right)$ offset reported in Fig. 2 clearly reveal the presence of a emission peak which shows increasing importance as the line excitation gets higher, suggesting the occurrence of a $\mathrm{SiO}$ clump tracing high excitation conditions and moving away from the driving source position.

Using the three observed $\mathrm{SiO}$ lines, it is possible to estimate the total column density $\left(N_{\mathrm{SiO}}\right)$ as well as the kinetic temperatures $\left(T_{\mathrm{k}}\right)$ and the hydrogen densities $\left(n_{\mathrm{H}_{2}}\right)$ by means of statistical-equilibrium calculations. We have used the Large Velocity Gradient (LVG) code we already taken into account for the $\mathrm{SiO}$ survey reported in Codella et al. (1999) and the collision rates given by Turner et al. (1992). The intensities of the spectra expressed in the mean-beam brightness temperature scale have been used, without correction due to the beam filling factor. As already discussed by Codella et al. (1999), if the $\mathrm{SiO}$ source sizes were definitely smaller than the three beamwidths, the LVG code would lead to an overestimate of the excitation conditions. In this case, the $T_{\mathrm{k}}, n_{\mathrm{H}_{2}}$ and $N_{\mathrm{SiO}}$ values could be reduced by factors of $1.2,2$ and 9 , respectively. On the other hand, if the $\mathrm{SiO}$ sources were definitely larger that the beamwidths, it would be better to use the spectra expressed in antenna temperature (corrected for atmospheric losses). In this case, the hydrogen densities would be reduced by a factor of up to 1.3 and the column densities by a factor of 2 .

The LVG results based on the $\left(0^{\prime \prime}, 0^{\prime \prime}\right)$ spectra lead either to a kinetic temperature of $\sim 50 \mathrm{~K}$ and a hydrogen density of about $3.0 \times 10^{6} \mathrm{~cm}^{-3}$ or to $T_{\mathrm{k}} \simeq 100 \mathrm{~K}$ and $n_{\mathrm{H}_{2}} \simeq 1.5 \times 10^{6} \mathrm{~cm}^{-3}$. Using the spectra of the SE1 clump, we have $T_{\mathrm{k}} \simeq 40 \mathrm{~K}(80 \mathrm{~K}), n_{\mathrm{H}_{2}} \simeq 4 \times 10^{6} \mathrm{~cm}^{-3}$ $\left(2 \times 10^{6} \mathrm{~cm}^{-3}\right)$. On the other hand, the data collected towards the SW1 position (where the SiO lines are definitely smaller) lead to $T_{\mathrm{k}} \simeq 20 \mathrm{~K}(40 \mathrm{~K})$ and $n_{\mathrm{H}_{2}} \simeq 3 \times 10^{6} \mathrm{~cm}^{-3}$ $\left(1.5 \times 10^{6} \mathrm{~cm}^{-3}\right)$. The $\mathrm{SiO}$ column densities are $2 \times 10^{13}$ (central map positions), $2 \times 10^{12} \mathrm{~cm}^{-2}$ (SE1 clump) and $4 \times 10^{12} \mathrm{~cm}^{-2}$ (SW1). These results confirm the association of the $\mathrm{SiO}$ molecule with high density gas at high temperatures.

\section{Dense cores and YSOs}

\subsection{High density cores identified in CS and $\mathrm{DCO}^{+}$maps}

The CS molecule is a well known tracer of high density material hosting Young Stellar Objects (YSOs) and has been already used to investigate the physical conditions in IC $1396 \mathrm{~N}$ by Serabyn et al. (1993), who performed a detailed multiline study. The present CS observations, based on a single transition, do not allow us to add information 


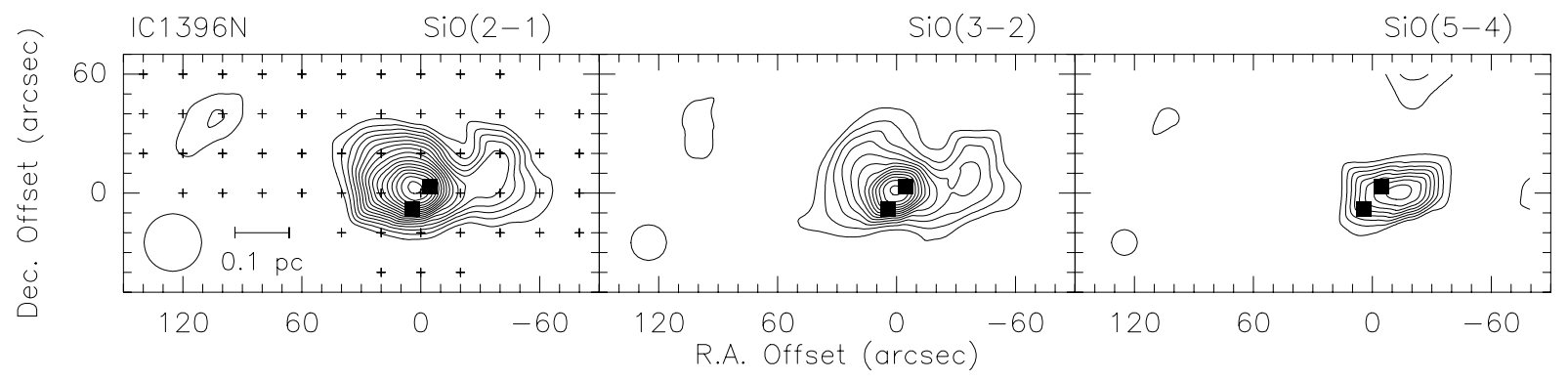

Fig. 5. Contour map of the $J=2-1, J=3-2$ and $J=5-4 \mathrm{SiO}$ (integrated) emission towards the IC $1396 \mathrm{~N}$ region. The velocity integration intervals are $-20,+50 \mathrm{~km} \mathrm{~s}^{-1}(J=2-1),-20,+40 \mathrm{~km} \mathrm{~s}^{-1}(J=3-2)$ and $-20,+20 \mathrm{~km} \mathrm{~s}-1(J=5-4)$. Symbols are drawn as in Fig. 1. The contour levels range from 0.69 to $8.05 \mathrm{~K} \mathrm{~km} \mathrm{~s}^{-1}$ (left panel), from 1.14 to $10.52 \mathrm{~K} \mathrm{~km} \mathrm{~s}^{-1}$ (center panel) and from 1.80 to $11.04 \mathrm{~K} \mathrm{~km} \mathrm{~s}^{-1}$ (right panel). The first contours and the steps correspond to $3 \sigma$ and $2 \sigma$, respectively.

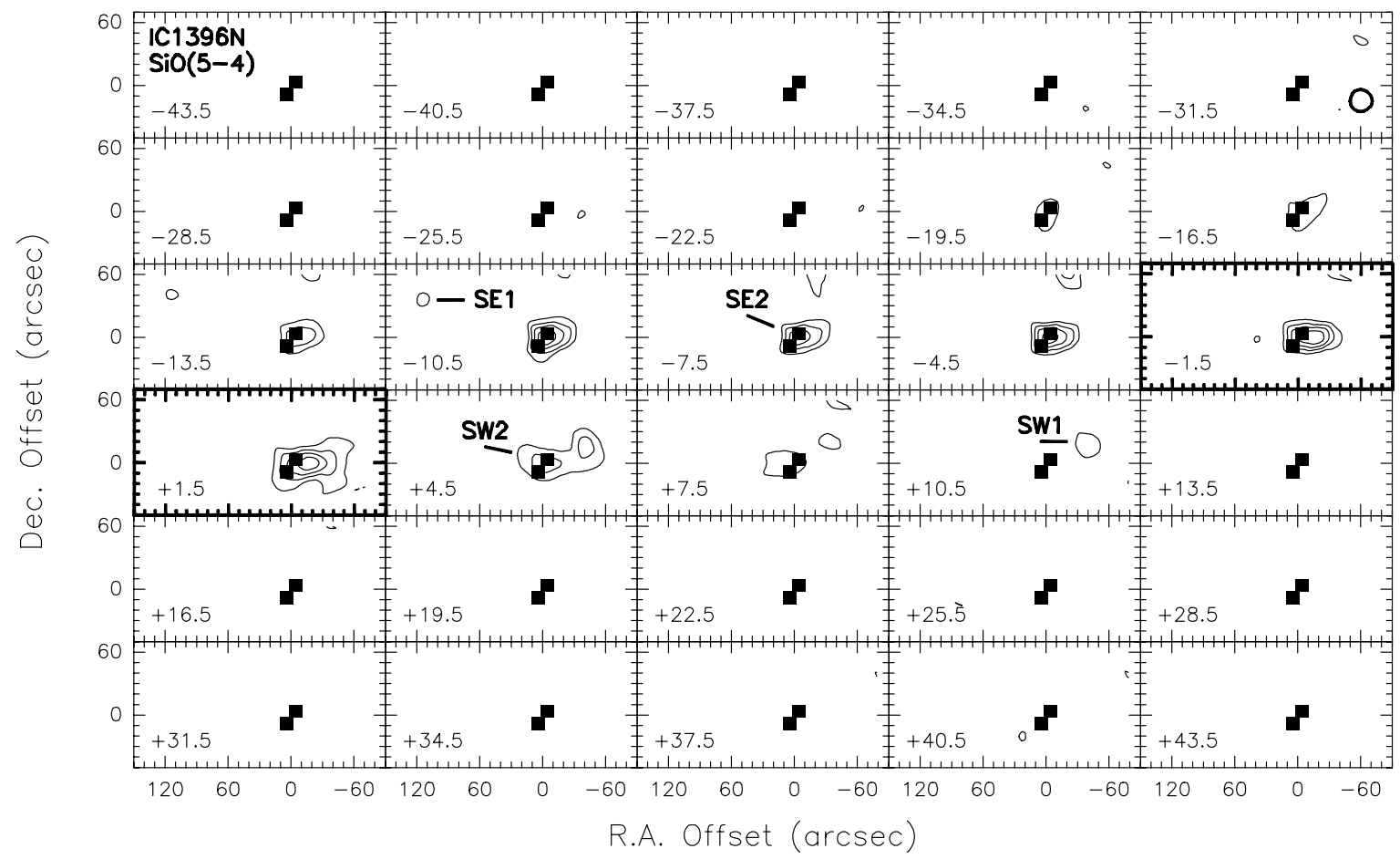

Fig. 6. Channel map of the $\mathrm{SiO} J=5-4$ emission towards IC 1396N. Each panel shows the emission integrated over a velocity interval of $3 \mathrm{~km} \mathrm{~s}^{-1}$ centred at the value given in the left corner. Symbols are drawn as in Fig. 3 . The contours range from 0.3 $(\sim 3 \sigma)$, to $1.2 \mathrm{~K} \mathrm{~km} \mathrm{~s}^{-1}$ by step of $0.3 \mathrm{~K} \mathrm{~km} \mathrm{~s}^{-1}$.

about the physical conditions associated with the CS emission with respect to that derived by Serabyn et al. (1993). However, here we present a high resolution $J=3-2$ map obtained with a better sampling and which explores for the first time the northern part of the globule, where the northern outflow has been discovered. The aim of the present CS observations is to have a plot of the distribution of the gas at velocities close to the ambient one and to try to indirectly detect the YSOs driving the bipolar outflows.

Figure 7 reports the CS $J=3-2$ channel map focusing the attention on the region around the $\left(0^{\prime \prime}, 0^{\prime \prime}\right)$ position. It is possible to see that a CS clump with a FWHM size of about $50^{\prime \prime}(0.18 \mathrm{pc})$ is located at the central position and at velocities around $0 \mathrm{~km} \mathrm{~s}^{-1}$, i.e. at the velocity and at the coordinates where the driving source is expected.
The CS profiles peak at an ambient LSR velocity between -0.5 and $+1.2 \mathrm{~km} \mathrm{~s}^{-1}$. However, Fig. 7 and the spectra reported in Fig. 2 show that the CS emission coming from the central region of IC $1396 \mathrm{~N}$ is not confined at the ambient velocity, but it is also able to trace higher velocities, up to $\sim \pm 10 \mathrm{~km} \mathrm{~s}^{-1}$, detecting the slower component of the outflow motion. Therefore, in order to definitely determine the ambient velocity, a map of $40^{\prime \prime} \times 40^{\prime \prime}$ centred at the $\left(0^{\prime \prime}, 0^{\prime \prime}\right)$ coordinates in the $\mathrm{DCO}^{+} J=2-1$ line has been obtained with a $0.21 \mathrm{~km} \mathrm{~s}^{-1}$ spectral resolution (Fig. 8). Actually, Bachiller \& Peréz Gutiérrez (1997) has shown that $\mathrm{DCO}^{+}$emission is one of the best tools to trace the gas condensations around protostars since its emission is not contaminated from outflow motions.

The $\mathrm{DCO}^{+}$spectra (see e.g. Fig. 2) show narrow lines $\left(F W H M \simeq 0.6-2.4 \mathrm{~km} \mathrm{~s}^{-1}\right)$, confirming that they can 


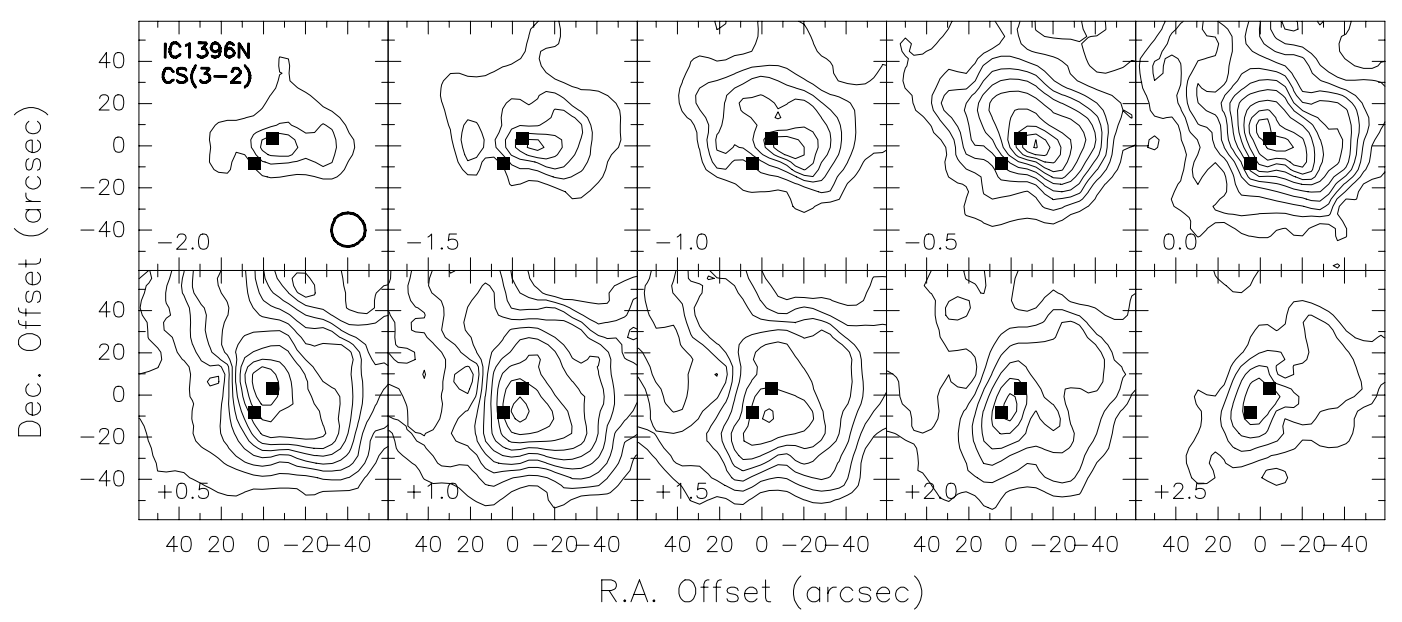

Fig. 7. Channel map of the CS $J=3-2$ emission towards the IC $1396 \mathrm{~N}$ central region. Each panel shows the emission integrated over a velocity interval of $0.5 \mathrm{~km} \mathrm{~s}^{-1}$ centred at the value given in the left corner. Symbols are drawn as in Fig. 3. The contours range from $0.4(\sim 5 \sigma)$, to $4.0 \mathrm{~K} \mathrm{~km} \mathrm{~s}^{-1}$ by step of $0.4 \mathrm{~K} \mathrm{~km} \mathrm{~s}^{-1}$.

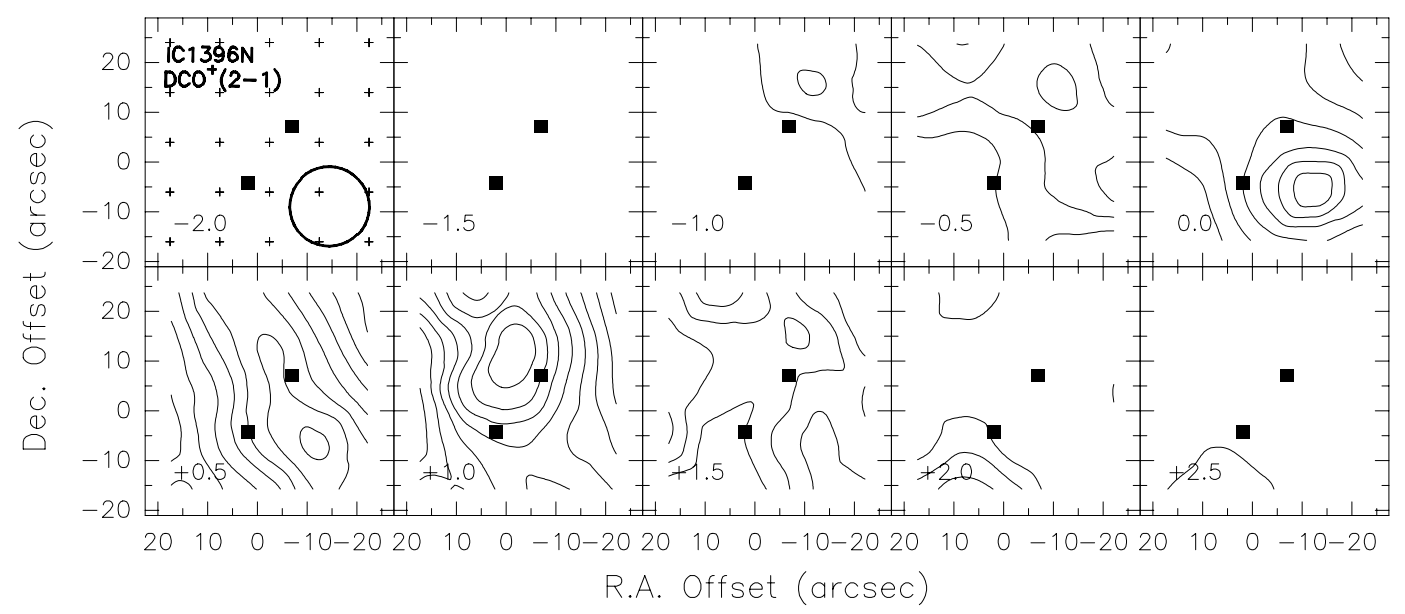

Fig. 8. Channel map of the $\mathrm{DCO}^{+} J=2-1$ emission towards IC $1396 \mathrm{~N}$. Each panel shows the emission integrated over a velocity interval of $0.5 \mathrm{~km} \mathrm{~s}^{-1}$ centred at the value given in the left corner. Symbols are drawn as in Fig. 3. The contours range from $0.21(\sim 3 \sigma)$, to $1.47 \mathrm{~K} \mathrm{~km} \mathrm{~s}^{-1}$ by step of $0.21 \mathrm{~K} \mathrm{~km} \mathrm{~s}^{-1}$.

be used as a tracer of the high density ambient gas. The $\mathrm{DCO}^{+}$channel map allows to detect three clumps with different velocities: at $\left(-10^{\prime \prime},+15^{\prime \prime}\right)$ and $-1 \mathrm{~km} \mathrm{~s}^{-1}$, at $\left(-10^{\prime \prime},-5^{\prime \prime}\right)$ and $0 \mathrm{~km} \mathrm{~s}{ }^{-1}$ and at $\left(0^{\prime \prime},+15^{\prime \prime}\right)$ and $+1 \mathrm{~km} \mathrm{~s}^{-1}$. Moreover, the maps partially detect a fourth clump with a velocity of about $+2 \mathrm{~km} \mathrm{~s}^{-1}$ and located in the southern direction with respect to the central coordinates. The clumps are not resolved by the $16^{\prime \prime}$ beamwidth and then their size is smaller than $0.07 \mathrm{pc}$. The present $\mathrm{DCO}^{+}$data are in agreement with the interferometric $\mathrm{C}^{18} \mathrm{O}$ map reported by Schwartz et al. (1991), where at least three compact structures are detected.

The $\mathrm{DCO}^{+}$observations clearly support the possibility that the IC $1396 \mathrm{~N}$ region hosts an aggregate of embedded YSOs, calling for a further analysis through continuum emission (see Sect. 4.2.1). Taking into account the geometry of the $\mathrm{CO}$ central outflow (see Fig. 3$)$, the $\left(0^{\prime \prime}\right.$, $\left.+15^{\prime \prime}\right) \mathrm{DCO}^{+}$structure seems the best candidate to be the clump with the driving source of the central outflow in its interior.
The situation seems simpler in the northern region of IC 1396 N. Figure 9 shows the CS $J=3-2$ channel map around the position of the northern outflow. We observe a clumpy structure, elongated along a roughly north-south direction, and $\simeq 45^{\prime \prime}(0.16 \mathrm{pc})$ wide. A well defined CS clump is centred on the $\left(-30^{\prime \prime},+140^{\prime \prime}\right)$ position, i.e. at the coordinates where the driving source has been detected (see Sect. 3.1).

The $\mathrm{CS}$ and $\mathrm{DCO}^{+}$total column densities have been calculated considering the temperature estimates derived form the $\mathrm{CO}$ data and using the spectroscopic constants given in the literature, e.g. by Bogey et al. (1981), Blake et al. (1987), Botschwina (1989), Swade (1989) and Lovas (1992). The CS observations of the central outflow, assuming a kinetic temperature of $20 \mathrm{~K}$, lead to $N_{\mathrm{CS}}$ $\simeq 2 \times 10^{14} \mathrm{~cm}^{-2}$ for the clump hosting the driving source, and to $\simeq 3-5 \times 10^{13} \mathrm{~cm}^{-2}$ for the positions along the outflow axis. On the other hand, the estimate of $N_{\mathrm{CS}}$ for the northern outflow, using a temperature of $10 \mathrm{~K}$, gives values between $8 \times 10^{12}$ and $2 \times 10^{13} \mathrm{~cm}^{-2}$. Finally, assuming 


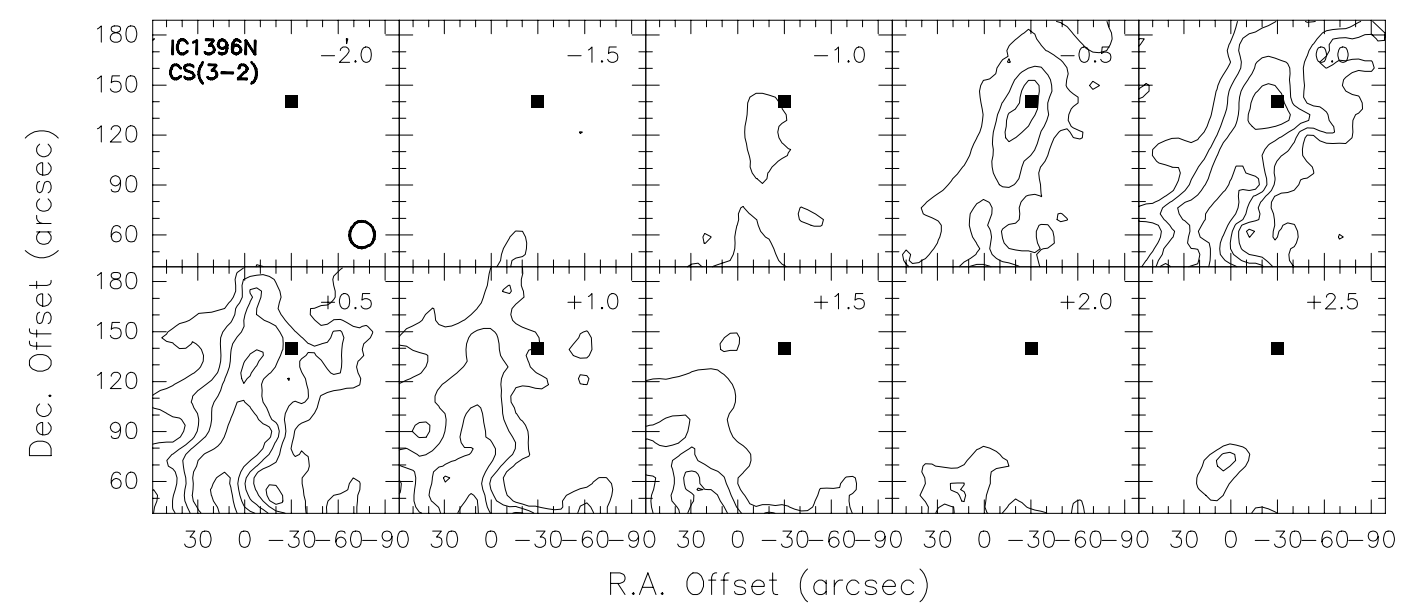

Fig. 9. Channel map of the CS $J=3-2$ emission towards the IC $1396 \mathrm{~N}$ northern outflow. Symbols and contours are drawn as in Fig. 7.

a kinetic temperature of $20 \mathrm{~K}$, total $\mathrm{DCO}^{+}$column densities of about $3 \times 10^{12} \mathrm{~cm}^{-2}$ have been derived for the clumps near the $\left(0^{\prime \prime}, 0^{\prime \prime}\right)$ position.

\subsection{The region around IRAS $21391+5802$}

\subsubsection{Stellar content}

The possibility that the central region of IC $1396 \mathrm{~N}$ hosts an aggregate of YSOs, as suggested in Sect. 4.1 by the $\mathrm{DCO}^{+}$observations, can be verified using Fig. 10, which shows the continuum interferometric maps at 88 (left panel), 92 (middle panel) and $110 \mathrm{GHz}$ (right panel). Two continuum unresolved sources have been detected at the three frequencies: we labelled A the most intense, which is located close to the IRAS coordinates, and B the fainter one, detected in the south-east direction. In Table 4 positions (given with an uncertainty of about 0.5 ) and integrated fluxes are reported. The source A had been already detected at $110 \mathrm{GHz}$ by Wilking et al. (1993) using the OVRO interferometer, whereas the component $\mathrm{B}$ is a new detection, thanks to the improved sensitivity of the array. The two millimeter components correspond, within the positional uncertainties, to the positions of two $\mathrm{H}_{2} \mathrm{O}$ maser sources as determined by Tofani et al. (1995) using the VLA accuracy of $\sim 0{ }^{\prime \prime} 1$. Thus, the outcoming scenario is that in the central region of IC $1396 \mathrm{~N}$ the star forming process occurs at least in two different places, separated by $14 . .5$, corresponding to about $10^{4} \mathrm{AU}$.

In Fig. 11 we show the spectral energy distribution (SED) of IC $1396 \mathrm{~N}$ from $6 \mathrm{~cm}$ to $12 \mu \mathrm{m}$, as obtained from our data (considering both $\mathrm{A}$ and $\mathrm{B}$ components) and from the literature (IRAS, Wilking et al. 1989a; Tofani et al. 1995; Saraceno et al. 1996). The portion of the SED from $60 \mu \mathrm{m}$ to $3.4 \mathrm{~mm}$ can be well fitted by a modifyed blackbody (Walker et al. 1990) with a temperature of $27 \mathrm{~K}$ and a power law dependence of the opacity coefficient with exponent $\beta=1.7$, hence the millimeter emission is well explained in terms of dust emission with little or no contribution from free-free emission from ionised gas, consistent with the non detections at centimeter wavelengths. It is worth noting that the temperature derived from the continuum emission is compatible with the gas temperature of 20-23 K derived by Serabyn et al. (1993) using the $\mathrm{NH}_{3}$ molecule. One should bear in mind that the various observations at different wavelengths have been collected using different angular resolutions. In particular, the radio continuum observations at $6,3.6,2$ and $1.3 \mathrm{~cm}$ (Wilking et al. 1989a; Tofani et al. 1995) have been made at the VLA, with synthesised beams always smaller than the separation between sources A and B; hence the upper limits are valid for both sources separately. On the other hand, the JCMT 1.3-0.35 mm observations (Saraceno et al. 1996) were made with $16^{\prime \prime}-19^{\prime \prime}$ beams centered on the nominal IRAS position and then they are mainly sensitive to the emission of component A, even if a contamination due to emission arising from the source B cannot be excluded (see the dotted circle in Fig. 10). Due to the large beams, the fluxes from IRAS observations contain the contributions from both sources. The excess emission at short wavelengths (IRAS 12 and $25 \mu \mathrm{m}$ ) is due to a warmer dust component or the integrated emission from embedded sources detected in the near IR (Nisini et al. 2001). Finally, it is worth noting that since the present $3 \mathrm{~mm}$ fluxes are consistent with the SED fit, which is essentially a fit of single dish data, indirectly we can say that the OVRO observations do not miss a considerable fraction of the continuum emission.

By integrating the continuum spectrum, we obtain an integrated luminosity of $233 L_{\odot}$, which is in good agreement with previous determinations (e.g. Wilking et al. 1989b; Saraceno et al. 1996 and references therein). However, we stress that this value of $233 L_{\odot}$ includes the whole region around the IRAS coordinates. In particular, an important contribution to the uncertainty associated with the estimation of the bolometric luminosity comes from the 60 and $100 \mu \mathrm{m}$ IRAS measurements, which are particularly sensitive to diffuse emission, and consequently, in the IC $1396 \mathrm{~N}$ case, to the emission due to the region of the globule heated by the external exciting star. Thus, the value of $233 L_{\odot}$ can be considered as a sort of 


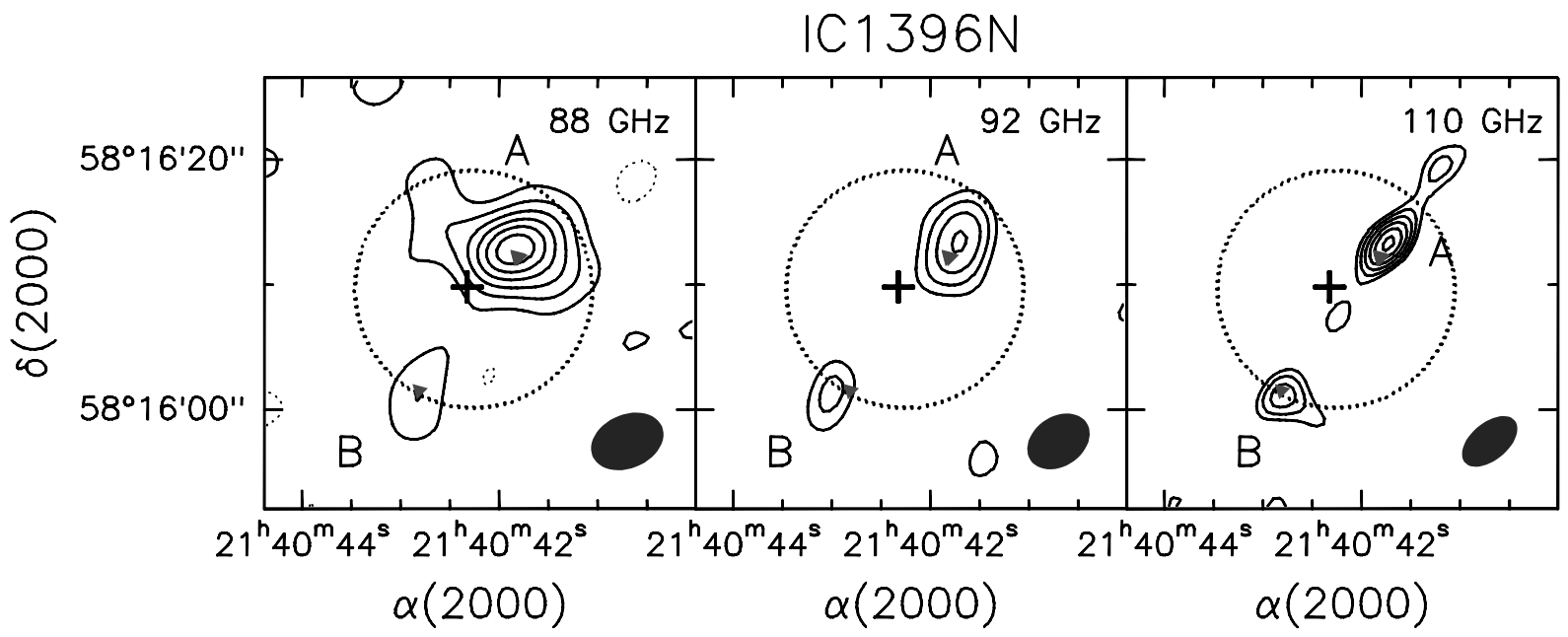

Fig. 10. Continuum maps at 88 (left panel), 92 (middle panel) and $110 \mathrm{GHz}$ (right panel) of the IC $1396 \mathrm{~N}$ central region. The contour levels are $-3.8,3.8$ to 18.8 by $3.0 \mathrm{mJy}^{-b_{e a m}{ }^{-1}}(88 \mathrm{GHz}),-7.5,7.5$ to 19.5 by $4.0 \mathrm{mJy}$ beam ${ }^{-1}(92 \mathrm{GHz})$ and $-20,20$ to 45 by $5 \mathrm{mJy}_{\text {beam }}^{-1}(110 \mathrm{GHz})$. The first contours correspond to about $3 \sigma$. The filled triangles stand for the positions of the $\mathrm{H}_{2} \mathrm{O}$ maser sources (Tofani et al. 1995), the dotted circle is for the JCMT beam used by Saraceno et al. (1996), the cross marks the IRAS coordinates, while the filled ellipse in the lower right corner shows the synthesised FWHM beam.

Table 4. Parameters of the continuum millimeter sources in the IC $1396 \mathrm{~N}$ central region.

\begin{tabular}{c|c|c|c|c|c}
\hline Source & \multicolumn{2}{|c|}{ Position } & \multicolumn{3}{c}{ Flux (mJy) } \\
& $\alpha(2000)$ & $\delta(2000)$ & $88 \mathrm{GHz}$ & $92 \mathrm{GHz}$ & $110 \mathrm{GHz}$ \\
\hline A & $21^{\mathrm{h}} 40^{\mathrm{m}} 41^{\mathrm{s}} .71$ & $+58^{\circ} 16^{\prime} 12^{\prime \prime} .8$ & $42 \pm 5$ & $42 \pm 6$ & $79 \pm 15$ \\
B & $21^{\mathrm{h}} 40^{\mathrm{m}} 42^{\mathrm{s}} .83$ & $+58^{\circ} 16^{\prime} 01^{\prime \prime} .4$ & $8 \pm 2$ & $11 \pm 3$ & $29 \pm 5$ \\
\hline
\end{tabular}

upper limit of the luminosities of the central continuum sources. On the other hand, the bolometric luminosity of the source driving the central molecular outflow (e.g. the A component, see Sect. 4.2.2) can be inferred also using the outflow dynamical parameters and the relationship given by Cabrit \& Bertout (1992). As already reported in Sect. 3.3, by assuming an inclination angle of $20^{\circ}$, we have about $3 L_{\odot}$, which can be used as a lower limit, since the obtained value is strongly dependent on the outflow geometry: values around $100 L_{\odot}$ can be derived taking $10^{\circ}$ as inclination angle.

Using the temperature and the power law dependence of the opacity coefficient calculated above it is possible to derive the mass of the dust condensations assuming optically thin thermal emission. We adopted a gas to dust ratio of 100 (by mass) and used the dust mass emissivity coefficient of $k_{230 \mathrm{GHz}}=0.005 \mathrm{~cm}^{2} \mathrm{~g}^{-1}$ as measured by Kramer et al. (1998) and scaled to the observed frequencies through $k_{\nu}=k_{230 \mathrm{GHz}}(\nu / 230)^{\beta}$. The masses derived at $88 \mathrm{GHz}$ for the $\mathrm{A}$ and $\mathrm{B}$ sources are $\simeq 20$ and $4 M_{\odot}$, respectively. The uncertainties, mainly due to the assumption of the $k_{230 \mathrm{GHz}}$ value, affect the values by a factor of $\sim 2$.

\subsubsection{Interferometric images of the outflow}

Figure 12 shows the $\mathrm{HCO}^{+} J=1-0$ spectra of the IC $1396 \mathrm{~N}$ central region. In the attempt to recover as much of the extended emission as possible without degrading the

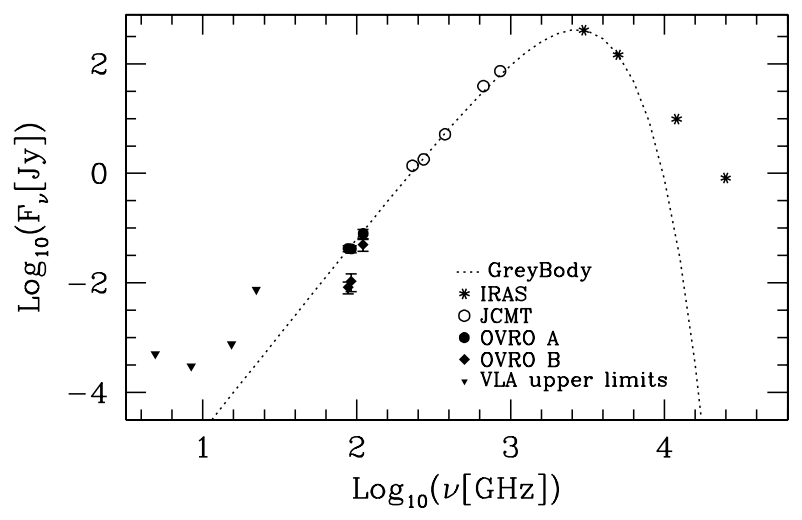

Fig. 11. Spectral energy distribution of IC $1396 \mathrm{~N}$ : see text for references.

resolution too much, we produced a data cube applying a $20 \mathrm{k} \lambda F W H M$ tapering Gaussian to the $(u, v)$ data. The resulting synthesised beam is approximately $9^{\prime \prime} \times 7^{\prime \prime}$. In Fig. 12 we show the low $\left(1.7 \mathrm{~km} \mathrm{~s}^{-1}\right)$ and high $\left(0.4 \mathrm{~km} \mathrm{~s}^{-1}\right)$ spectral resolution spectra obtained from the data cubes by integrating each plane over the whole area of $\mathrm{HCO}^{+}$ emission. Several kinematic features have been marked by detecting high velocity wings and the component at velocities close to the ambient ones resulting from the 30-m observations (see Sect. 4.1). The broad red and blue wings have been separated into two velocity regimes: the low velocity (from -11.5 to $-4.5 \mathrm{~km} \mathrm{~s}^{-1}$ and from +5.3 to $+12.2 \mathrm{~km} \mathrm{~s}^{-1}$, for the blue- and redshifted gas, respectively) and the high velocity $\left(-48.0\right.$ to $-11.5 \mathrm{~km} \mathrm{~s}^{-1}$ 


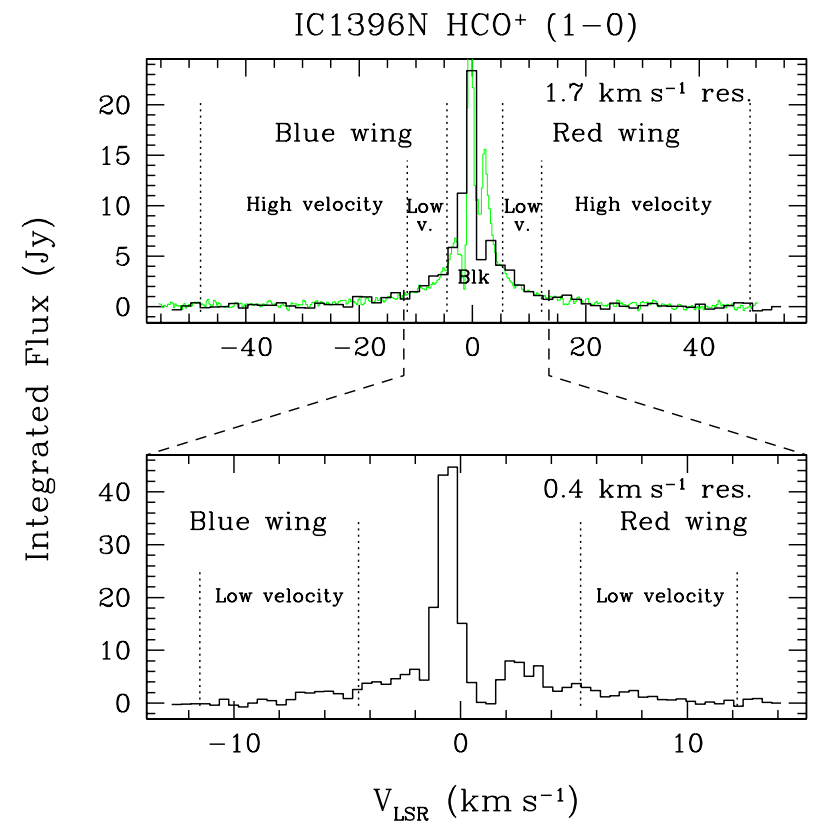

Fig. 12. Low $\left(1.7 \mathrm{~km} \mathrm{~s}^{-1}\right.$; upper panel) and high spectral resolution $\left(0.4 \mathrm{~km} \mathrm{~s}^{-1}\right.$; lower panel) $\mathrm{HCO}^{+} J=1-0$ spectra obtained with the OVRO array and calculated integrating over the whole emitting region in IC $1396 \mathrm{~N}$. In the upper panel the 30-m IRAM spectrum is reported in gray (Cesaroni et al. 1999). The velocity features discussed in the text (see Sect. 4.2.2) are reported: the dotted lines bound the velocity range of the blue and red wings.

and from +12.2 to $+49.0 \mathrm{~km} \mathrm{~s}^{-1}$ ). The hereafter called bulk emission, defined in the low resolution spectrum as the emission from -4.5 to $+5.3 \mathrm{~km} \mathrm{~s}^{-1}$, is shown in the high resolution spectrum. It is possible to detect a doublepeak profile in agreement with the single-dish (IRAM $30-\mathrm{m}) \mathrm{HCO}^{+} J=1-0$ spectrum reported by Cesaroni et al. (1999) and drawn in gray in Fig. 12 (upper panel). The comparison between the OVRO and the $30-\mathrm{m} \mathrm{HCO}^{+}$ spectra allows us to check how much flux is lost by the interferometric observations because of extended emission. Figure 12 clearly shows that the two spectra are in a perfect agreement for the low and high velocity wings. This ensures us that we are missing no component (located inside the $30-\mathrm{m}$ beam) of the $\mathrm{HCO}^{+}$outflow, whose analysis represents the aim of the OVRO line observations. On the other hand, Fig. 12 shows that at ambient velocities the 30-m observations detect emission which is lost by the OVRO interferometer, suggesting the occurrence of an extended envelope.

Figure 13 reports the $\mathrm{HCO}^{+}$integrated low (upper panel) and high (lower panel) velocity wings emission towards IC $1396 \mathrm{~N}$, with superimposed the $\mathrm{H}_{2}(1-0) \mathrm{S}(1) \mathrm{im}-$ age obtained by Testi \& Felli (private communication) with the ARNICA camera mounted on the TIRGO $1.5 \mathrm{~m}$ telescope. Both the low and high velocity wings show a bipolar structure centered on the continuum source A, clearly indicating that such object is the driving source of the central CO outflow. The low velocity gas shows a substantial superposition of the blue- (gray contours) and

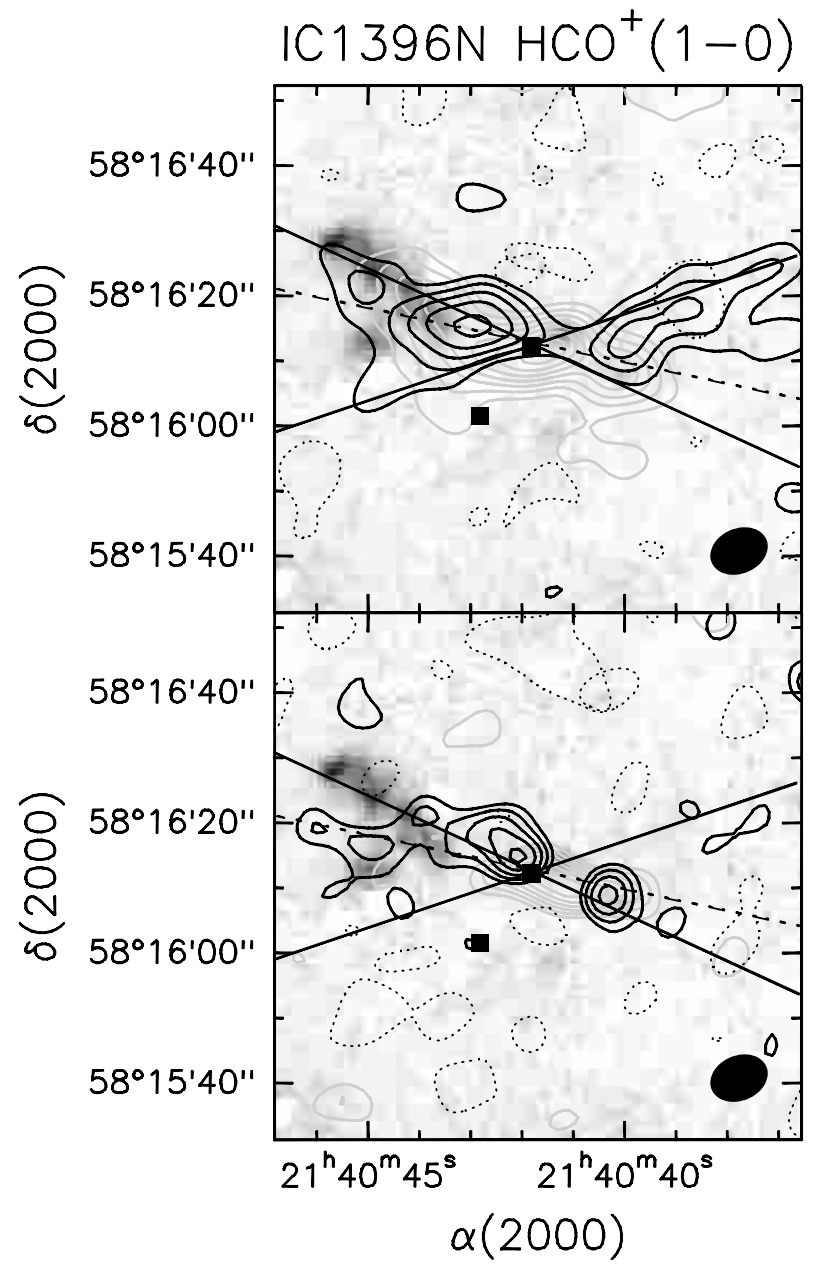

Fig. 13. Contour map of the $\mathrm{HCO}^{+} J=1-0$ outflow towards IC $1396 \mathrm{~N}$ with superimposed the $\mathrm{H}_{2}(1-0) \mathrm{S}(1)$ image obtained by Testi \& Felli (private communication). Black and gray contours are for the red and blue emission, respectively. The top panel reports the low velocity outflow $\left(-11.5\right.$ to $-4.5 \mathrm{~km} \mathrm{~s}^{-1}$ and +5.3 to $+12.2 \mathrm{~km} \mathrm{~s}^{-1}$ ), while the bottom panel shows the high velocity component $\left(-48.0\right.$ to $-11.5 \mathrm{~km} \mathrm{~s}^{-1}$ and +12.2 to $+49 \mathrm{~km} \mathrm{~s}^{-1}$ ). The filled ellipse shows the synthetised $F W H M$ beam. The solid lines mark the low velocity biconical structure, the dot-dashed lines shows the approximate direction of the high velocity gas (see Sect. 4.2.2), while the squares mark the A and B continuum components. Contour levels for the low velocity (blue and red) outflow are $-0.6,0.6$ to $3.6 \mathrm{Jy} \mathrm{km} \mathrm{s}^{-1}$ beam $^{-1}$. Contour levels for the high velocity outflow are -1.6 , 1.6 to $4.1 \mathrm{Jy} \mathrm{km} \mathrm{s}^{-1}$ beam $^{-1}$ for the blue lobe and $-1.2,1.2$ to $3.2 \mathrm{Jy} \mathrm{km} \mathrm{s}^{-1}$ beam $^{-1}$ for the red emission. First contours correspond to $\sim 2 \sigma$, while the steps are $0.5 \mathrm{Jy} \mathrm{km} \mathrm{s}^{-1}$ beam $^{-1}$.

redshifted (black) components, confirming the present $\mathrm{CO}$ and $\mathrm{SiO}$ results (Sect. 3). A biconical structure, pointed out by the solid lines in Fig. 13, is tentatively detected. It is also possible to see that the western red component bends towards the north in agreement with the single-dish CO observations.

On the other hand, the high velocity gas appears to be well collimated into two wiggling unresolved $(\leq 0.02 \mathrm{pc})$ plumes emerging from the central object roughly within the conical structure defined by the low velocity gas, and 


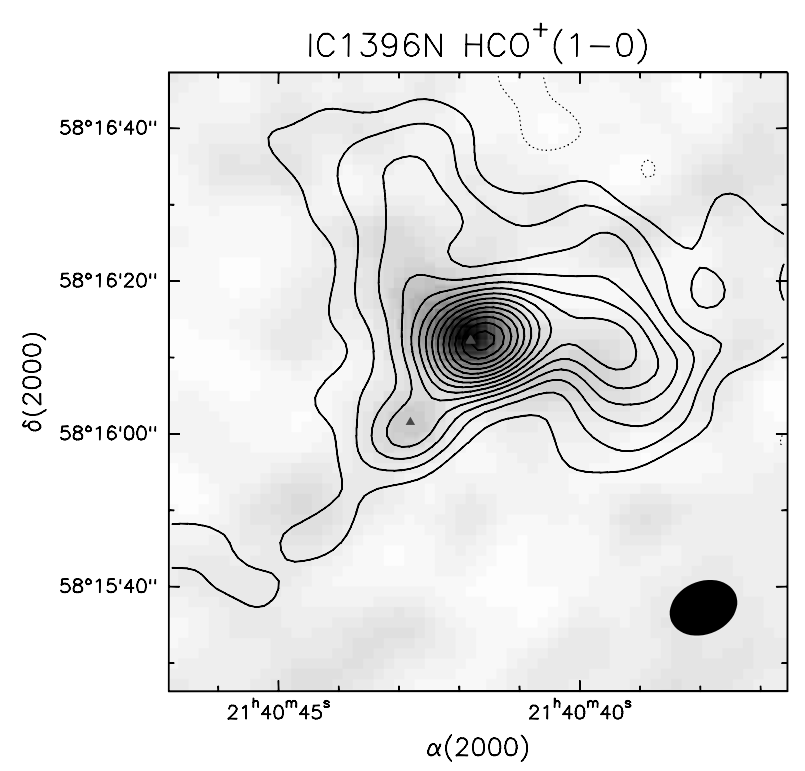

Fig. 14. Contour map of the $\mathrm{HCO}^{+} J=1-0$ bulk (from -4.5 to $+5.3 \mathrm{~km} \mathrm{~s}^{-1}$ ) emission (see Sect. 4.2 .2 ) towards IC $1396 \mathrm{~N}$ with superimposed the $88 \mathrm{GHz}$ continuum image. Symbols are drawn as in Fig. 10. The contour levels are $-1.5,1.5$ to $21.0 \mathrm{Jy} \mathrm{km} \mathrm{s}^{-1}$ beam $^{-1}$, with a step of $1.5 \mathrm{Jy} \mathrm{km} \mathrm{s}^{-1}$ beam $^{-1}$ and corresponds to about $2 \sigma$.

along the direction marked in Fig. 13 by the dot-dashed line. The two high velocity lobes are better separated with respect to the lower velocity components. Figure 13 shows that the redshifted gas emerges to the east, whereas the blue component extends to the west, even if a red clump is also clearly detected in such direction. It is worth noting that the eastern redshifted high velocity component detected with the OVRO interferometer seems to be in contradiction with the high velocity jet-like structure detected to the east with the 30-m antenna at a definitely larger distance from the continuum source A (SE1 clump, see Fig. 3). However, this apparent disagreement, together with the overlap of the red- and blueshifted lobes, can be explained by the small inclination to the plane of sky, as deduced also by the $30-\mathrm{m}$ results, and by the interaction of the outflow with the ambient medium. Another solution could be found by assuming that the outflow axis has changed its direction from the period in which the driving source produced the SE1 and SW1 CO clumps to the most recent ejection events, traced by the $\mathrm{HCO}^{+}$ emission close to the source A. The change of the direction of the axis could be due to precession as well as to a deflection caused by the surrounding medium, but, unfortunately, the present data do not allow us to discuss these possibilities.

Finally, Fig. 13 shows that, in agreement with the CO and $\mathrm{SiO}$ observation, the western lobe of the high velocity $\mathrm{HCO}^{+}$outflow is less extended, as explained by the occurrence of high density material along such direction. In particular (see also Nisini et al. 2001), no $\mathrm{H}_{2}$ emission is observed, probably due to high extinction.

Figure 14 reports the contour maps of the integrated bulk $\mathrm{HCO}^{+} J=1-0$ emission towards IC $1396 \mathrm{~N}$. A quite elongated clump is detected: its size is $\simeq 40^{\prime \prime}(0.15 \mathrm{pc})$ and coincides with the CS clump detected at the $30-\mathrm{m}$ antenna. The $\mathrm{HCO}^{+}$clump seems to be fragmented in several substructures, the most intense of them peaking close to the continuum source A.

\section{Evolution of the cometary globule}

The impinging of external radiation on a molecular globule, due to a high mass star located in its neighbourhood, has been shown to cause the globule implosion. This is known as the Radiative Driven Implosion (RDI) mechanism (Reipurth 1983). Bertoldi (1989) and Bertoldi \& McKee (1990) developed a model in which this process occurs in two phases: first the globule is compressed by the ionizing radiation and then it assumes the head-tail morphology. Later on, Lefloch \& Lazareff (1994, 1995) produced a dynamical model which has been successfully applied to several cometary globules observed to date and which foresees a collapse and transient phase, tending to a quasi-stationary equilibrium, followed by the classical cometary phase, in which the globule continues to be accelerated and photo-evaporated.

The collapse and transient phase lasts $\sim 10^{5} \mathrm{yr}$ and it is spectroscopically characterized by blue and red wings which follow the expansions and the re-compressions of the globule. The cometary regime, whose lifetime is typically $10^{6} \mathrm{yr}$, is characterized only by a weak overall velocity gradient. The occurrence of several molecular outflows does not allow to study in details the kinematics of the globule hosting the star forming process and, in particular, does not allow to do a clear association between the spectroscopic signatures and the evolutionary stage of IC $1396 \mathrm{~N}$. For instance, as already reported in Sect. 3.3, there is a close interaction of the outflow motion with the ambient medium and the outflow structure can already be identified at low velocities. However, the IC $1396 \mathrm{~N}$ CO channel maps reported in Fig. 3 show that the low velocity panels $\left(\sim \pm 6 \mathrm{~km} \mathrm{~s}^{-1}\right)$ clearly trace the rim-shape structure of the southern part of the globule. In addition, there is no clear indication of velocity gradient along the major axis of the cloud. The detection of blue- and redshifted emission suggests that IC $1396 \mathrm{~N}$ is in the collapse and transient phase, undergoing one of the expansions or compressions described in the model of Lefloch \& Lazareff (1994). This indication is also supported by the short kinematical timescales derived for the CO outflows $\left(\leq 3 \times 10^{4} \mathrm{yr}\right)$, which confirm the youth of the stellar objects inside the globule.

Following Lefloch \& Lazareff (1994) and Lefloch et al. (1997), the mass loss rate of the cloud induced by photoionization of the external layers is:

$$
\dot{M}=29\left(\frac{\Phi}{10^{7} \mathrm{~cm}^{-2} \mathrm{~s}^{-1}}\right)^{1 / 2}\left(\frac{R}{1 \mathrm{pc}}\right)^{3 / 2} M_{\odot} \mathrm{Myr}^{-1}
$$

where $\Phi=N_{\mathrm{L}} / 4 \pi d^{2}$ is the ionizing flux, $N_{\mathrm{L}}$ is the total flux of Ly-c photons $(\geq 13.6 \mathrm{eV})$ in photons $\mathrm{s}^{-1}, d$ is the distance of the ionizing star from the globule and $R$ is 


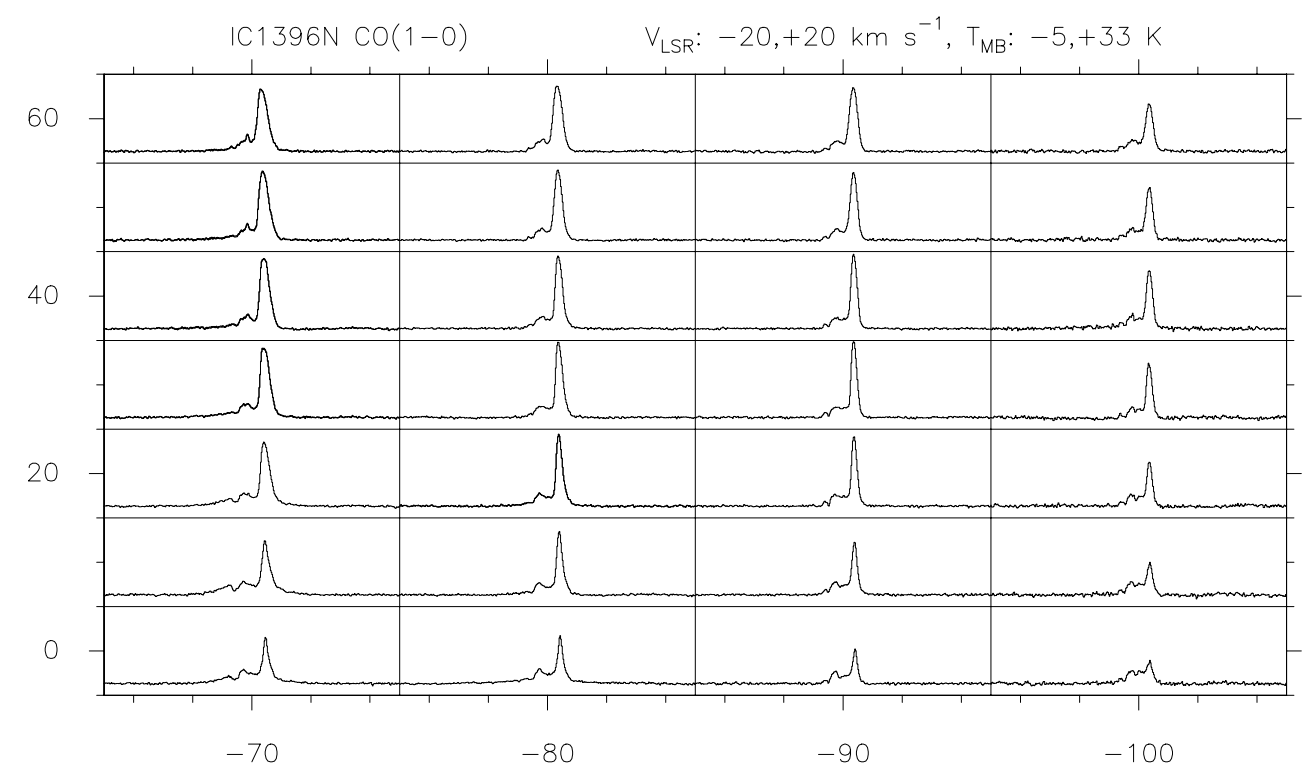

Fig. 15. CO $J=1-0$ spectra of the IC $1396 \mathrm{~N}$ west region. The horizontal and vertical scales correspond to RA and Dec Offset in arcseconds, respectively. The velocity range is from -20 to $+20 \mathrm{~km} \mathrm{~s}^{-1}$, while the $T_{\mathrm{MB}}$ scale lies between -5 and $+33 \mathrm{~K}$ and has been chosen accordingly to the strongest peak, associated with the $\left(-80^{\prime \prime},+30^{\prime \prime}\right)$ offset.

the size of the cloud. By applying the model to IC $1396 \mathrm{~N}$, we have $N_{\mathrm{L}}=6.6 \times 10^{48}$ photons s ${ }^{-1}$ (O6.5 type star), $d=11 \mathrm{pc}, \Phi=4.7 \times 10^{8} \mathrm{~s}^{-1} \mathrm{~cm}^{-2}$ and $R \simeq 0.7 \mathrm{pc}$ (see Fig. 1) for a mass loss rate of about $120 M_{\odot} \mathrm{Myr}^{-1}$.

Another result provided by the $\mathrm{CO}$ observations is the detection of an intense and narrow component around the $\left(-80^{\prime \prime},+30^{\prime \prime}\right)$ offset. This effect is visible in Fig. 3 , where, in the $+1.5 \mathrm{~km} \mathrm{~s}^{-1}$ panel, it is possible to see an elongated structure not detected at other velocities. The CO $J=$ $2-1$ component peaks at about $45 \mathrm{~K}$ and $+1.6 \mathrm{~km} \mathrm{~s}^{-1}$, with a linewidth $(F W H M)$ between 0.7 and $0.9 \mathrm{~km} \mathrm{~s}^{-1}$. Figure 15 reports the CO $J=1-0$ spectra which refer to such part of IC $1396 \mathrm{~N}$. The main line is located at the same velocity of the $J=2-1$ one, its linewidth lies between 0.8 and $1.0 \mathrm{~km} \mathrm{~s}^{-1}$ and the $T_{\mathrm{MB}}$ peaks at about $33 \mathrm{~K}$. This effect, occurring in one of the part of the globule facing the HD 206267 star, can be due to an enhanced heating of gas. The present result has been compared with theoretical calculations by Hollenbach et al. (1991), who presented models of photodissociation regions (PDRs), i.e. the predominantly neutral outer layers of molecular clouds exposed to an external far-ultraviolet (FUV) radiation field ranging from 6 and $13.6 \mathrm{eV}$. The incident FUV flux $\left(G_{0}\right)$ measured in units of the equivalent average local interstellar flux $\left(1.6 \times 10^{3} \mathrm{ergs} \mathrm{cm}^{-2} \mathrm{~s}^{-1}\right.$; Habing 1968) can be calculated through (Sternberg \& Dalgarno 1989):

$G_{0}=170 \frac{L}{L_{\odot}}\left(\frac{d}{1 \mathrm{pc}}\right)^{-2}\left(T_{4}\right)^{-4} \frac{1}{\mathrm{e}^{14.4 / T_{4}}-1}$

where $L$ and $T_{4}$ are the star luminosity and temperature, in units of $10^{4} \mathrm{~K}$, respectively. In the case of HD 206267, we have $L=10^{5.2} L_{\odot}$ and $T_{4}=4$ (Panagia 1973), leading to $G_{0} \simeq 24$. In case of a spherical H II region, produced by an $\mathrm{O}$ star, in equilibrium with the surrounding neutral gas, the density and the FUV incident flux are related by $G_{0} \simeq 10^{2}\left(n_{\mathrm{H}_{2}} / 10^{3} \mathrm{~cm}^{-3}\right)^{1.33}$ (Tielens \& Hollenbach 1985). This provides an estimate of the density: $\sim 340 \mathrm{~cm}^{-3}$. With these $G_{0}$ and $n_{\mathrm{H}_{2}}$ estimations and taking the $T_{\mathrm{MB}}$ peaks of the $\mathrm{CO}$ component as a rough estimate of the local gas temperature, it is possible to infer from the model of Hollenbach et al. (1991) a visual extinction of about $A_{\mathrm{v}} \leq 3$. If so, the IC $1396 \mathrm{~N}$ region traced by the $\mathrm{CO}$ component at $+1.6 \mathrm{~km} \mathrm{~s}^{-1}$ should trace the cool $(T \simeq 50 \mathrm{~K})$ part of a dense PDR, at $A_{\mathrm{v}} \sim 2-5$, where the gas is partially dissociated and warmed up also by the grain photoelectric heating, and where one of the main gas cooling terms in the energy balance is the $\mathrm{CO}$ emission.

The idea that in IC $1396 \mathrm{~N}$ star formation could be due to the occurrence of the RDI process has been suggested by Sugitani et al. (1989), with the first detection of the central molecular outflow inside the globule. Later on, in their study of the high density material located in IC 1396 N, Serabyn et al. (1993) claimed that the ionization front has only compressed the outermost layers of the cloud. The fact that the CS peak lies close to the IRAS coordinates and not along the bright rim induced the authors to believe that the globule had already evolved towards collapse on its own. Moreover, they estimated that the shock running into the cloud is relatively weak, with velocity of about $0.6 \mathrm{~km} \mathrm{~s}^{-1}$, which cannot be very effective in compressing the gas. On the other hand, Sugitani et al. (1995) through a near IR survey of bright rimmed clouds found that in a number of cases the bluer and older stars embedded in the clouds are located closer to the external exciting star than the redder and younger embedded ones, suggesting sequential star formation. Another example of 
cometary globule where star formation could be triggered by photo-ionization is IC 1848, according to Lefloch et al. (1997).

The present observations do not allow to clarify if the star forming processes in IC $1396 \mathrm{~N}$ are triggered by the advance of the shock caused by the UV radiation from the exciting star. However, they draw a new scenario regarding the stellar content of the globule pointing out that the star formation activity occurs also in the northern part of IC $1396 \mathrm{~N}$, at $0.5 \mathrm{pc}$ from the central CS peak. In summary, the present results show that at least two star forming sites are located in the central region of the globule, around the IRAS coordinates. Two mm-wavelength sources have been detected: one is the driving source of the central outflow, with a dynamical age of $2-3 \times 10^{4} \mathrm{yr}$, whereas the other source is apparently associated with no strong outflow process. Moreover, in the northern part of the globule another YSO has been detected, driving an outflow which has been found about $2 \times 10^{4}$ yr old. Between the central and the northern outflows, blue- and redshifted CO wings gas as well as $\mathrm{H}_{2}$ jet-like structures (Nisini et al. 2001) could indicate the presence of additional stellar objects, although in this case we cannot rule out the possibility that the wing emission arises from photo-evaporating gas at the surface of the globule. Taking into account the structure of the CS map (Fig. 1), the present data suggest that the star formation activity mainly occurs throughout the high density structures roughly located along the southnorth direction, which is similar to that determined by the IRAS 21391+5802-HD 206267 coordinates.

\section{Conclusions}

The bright rimmed globule IC $1396 \mathrm{~N}$, excited by the O6.5 star HD 206267 located in the Cep OB2 association, has been investigated through a multiline survey at mmwavelengths using the 30-m IRAM antenna and the OVRO interferometer. Emission due to $\mathrm{CO}, \mathrm{CS}, \mathrm{HCO}^{+}$, $\mathrm{DCO}^{+}$and $\mathrm{SiO}$ has been observed, allowing to trace different physical conditions inside the globule. Moreover, continuum emission at 88, 92 and $110 \mathrm{GHz}$ has been studied through interferometric observations, in order to investigate the stellar content of IC $1396 \mathrm{~N}$. The main results are the following:

1. The high resolution $\mathrm{CO}$ and CS observations confirm the cometary nature of the globule revealing its headtail structure with a high density elongated CS component ( $0.8 \mathrm{pc}$ long) pointing towards the exciting star. IC $1396 \mathrm{~N}$ is associated with at least two molecular outflows, both with a quite small inclination on the plane of the sky (in the $10^{\circ}-20^{\circ}$ range): one located around the IRAS coordinates (called central outflow) and another one, detected for the first time, located in the northern part of the globule (northern outflow). The driving sources have been individuated through the observation of continuum emission. Moreover, the detection of blue- and redshifted gas throughout the
CS structure suggest the occurrence of other outflows which have not been clearly individuated because the complexity of the region.

2. The central outflow is associated with an excitation temperature of $10-20 \mathrm{~K}$ and a total mass of $0.1 M_{\odot}$ equally distributed between the red and blue lobes. The estimation of the kinematical parameters such as the momentum, the kinetic energy and the mechanical luminosity is strongly affected by the uncertainty associated with the assumption of the angle of inclination on the plane of the sky. We have about $2-4 M_{\odot} \mathrm{km} \mathrm{s}^{-1}, 5 \times 10^{44}-2 \times 10^{45} \mathrm{erg}$ and $0.2-1.2 L_{\odot}$, respectively. Since the central outflow main structure is already detectable at low velocities $\left( \pm 3 \mathrm{~km} \mathrm{~s}^{-1}\right)$, there is indication that a considerable fraction of the $\mathrm{CO}$ emission is due to material interacting with the mass loss process. On the other hand, the northern outflow is less massive, $0.01 M_{\odot}$, while the kinetic energy is about $2 \times 10^{44} \mathrm{erg}$ and the luminosity $0.19 L_{\odot}$. The bolometric luminosities of the two sources driving the two outflows, as inferred from the $\mathrm{CO}$ data, are $\sim 4 L_{\odot}$ (northern) and 3-100 $L_{\odot}$ (central).

3. The CS observations allow to detect the high density molecular clumps hosting the driving sources of the two molecular outflows. In the central region of IC $1396 \mathrm{~N}$ the clump size is about $0.18 \mathrm{pc}$, while in the direction of the northern outflow, the clump is located inside a quite elongated structure $\simeq 0.16 \mathrm{pc}$ wide. The CS column density is $\sim 2 \times 10^{14}$ and $10^{13} \mathrm{~cm}^{-2}$ for the central and the northern clump, respectively. The distribution of the ambient material of the central region has been investigated also using $\mathrm{DCO}^{+}$emission. The emerging scenario is quite complex: at least three unresolved $(\leq 0.07 \mathrm{pc})$ clumps have been detected at different velocities in the same region traced by CS. The typical $\mathrm{DCO}^{+}$column density is about $3 \times 10^{12} \mathrm{~cm}^{-2}$.

4. By using $\mathrm{SiO}$ as a tracer of the high velocity central outflow components, a jet-like structure located along the main axis has been detected. The existence of four clumps, observed also thanks to the $\mathrm{CO}$ emission, reveal that episodic increases of the mass loss process have occurred. The size of the clumps is $\leq 0.1 \mathrm{pc}$, while the collimation factor is about 6 . The $\mathrm{SiO}$ emission presents different linewidths in different map positions, ranging from broad profiles $\left(25-30 \mathrm{~km} \mathrm{~s}^{-1}\right.$ for the $J=2-1$ component) to quite narrow lines $\left(5 \mathrm{~km} \mathrm{~s}^{-1}\right)$. The narrow emission can be interpreted as a result of the interaction between the outflow and the neighbouring high density medium: the shocked gas, produced at high velocities slows down loosing momentum. By means of statistical-equilibrium calculations, using a LVG code, we have estimated kinetic temperatures of $\sim 50-100 \mathrm{~K}$ and hydrogen densities of fews $10^{6} \mathrm{~cm}^{-3}$. The $\mathrm{SiO}$ column densities lie between $10^{12}$ and $10^{13} \mathrm{~cm}^{-2}$.

5. Interferometric continuum observations at 88, 92 and $110 \mathrm{GHz}$ of the central region of IC $1396 \mathrm{~N}$ show two continuum unresolved sources. The source labelled A 
is the most intense one and lies close to the IRAS coordinates, while the fainter one, called $\mathrm{B}$, is located at about $10^{4} \mathrm{AU}$ in the south-east direction. The two millimeter components correspond to the positions of two $\mathrm{H}_{2} \mathrm{O}$ maser sources and indicate that the star forming process occurs at least in two different places in the area around the IRAS coordinates. The masses derived at $88 \mathrm{GHz}$ for the $\mathrm{A}$ and $\mathrm{B}$ sources are about 20 and $4 M_{\odot}$, respectively.

6. The $\mathrm{HCO}^{+}$OVRO observations reveal several kinematic features in the central region. On the one hand, high velocity wings trace a bipolar highly collimated molecular outflow centered on the continuum source A, clearly indicating that such object is the driving source of the mass loss process detected with the $30-\mathrm{m}$ antenna and associated with the central outflow. No outflow has been detected towards the source B. On the other hand, the emission close to the ambient LSR velocity traces an elongated clump, with a size of about $0.15 \mathrm{pc}$, which coincides with the CS clump observed with the 30-m antenna.

7. The CO channel maps show that the rim-shape structure of the globule is clearly traced at low velocities, $\leq \pm 6 \mathrm{~km} \mathrm{~s}^{-1}$. The detection of such blue- and redshifted emission suggests that IC $1396 \mathrm{~N}$ is in the collapse and transient phase, undergoing one of the expansions or compressions foreseen by the model of Lefloch \& Lazareff (1994) for the earliest evolutionary stages of a cometary globule. Moreover, the present observations indicate that the star forming process is occurring also in the northern part of IC $1396 \mathrm{~N}$, at $0.5 \mathrm{pc}$ from the central CS peak.

Acknowledgements. We wish to thank P. Caselli and M. Tafalla for helpful discussions and suggestions. We are grateful to M. Tafalla also for his help during the observation performed using the MPIfR bolometer. The Owens Valley millimeter-wave array is supported by NFS grant AST-96-13717. Research at Owens Valley on the formation of young stars and planets is also supported by the Norris Planetary Origins Project.

\section{References}

Bachiller, R., \& Peréz Gutiérrez, M. 1997, ApJ, 487, L93

Bachiller, R., \& Tafalla, M. 1999, in The Origin of Stars and Planetary Systems, ed. C. J. Lada, \& N. D. Kylafis (Kluwer, Dordrecht), 227

Bertoldi, F. 1989, ApJ, 346, 735

Bertoldi, F., \& McKee, C. F. 1990, ApJ, 354, 529

Blake, G. A., Sutton, E. C., Masson, C. R., \& Phillips, T. G. 1987, ApJ, 316, 45

Bogey, M., Demuynck, C., \& Destombes, J. L. 1981, Mol. Phys., 43, 1043

Bontemps, S., André, P., Terebey, S., \& Cabrit, S. 1996, A\&A, 297, 98

Botschwina, P. 1989, in Ion and Cluster Spectroscopy and Structure, ed. J. P. Maier (New York, Elsevier), 59

Cabrit, S., \& Bertout, C. 1992, A\&A, 261, 274

Cesaroni, R., Felli, M., \& Walmsley, C. M. 1999, A\&AS, 136, 333
Codella, C., Bachiller, R., \& Reipurth, B. 1999, A\&A, 343, 585

Habing, H. J. 1968, Bull. Astr. Inst. Netherlands, 19, 421

Hollenbach, D. J., Takakashi, T., \& Tielens, A. G. G. M. 1991, ApJ, 377, 192

Kramer, C., Alves, J., Lada, C., et al. 1998, A\&A, 329, L33

Lada, C. J. 1985, ARA\&A, 23, 267

Lada, C. J., \& Fich, M. 1996, ApJ, 459, 638

Lefloch, B., \& Lazareff, B. 1994, A\&A, 289, 559

Lefloch, B., \& Lazareff, B. 1995, A\&A, 301, 522

Lefloch, B., Lazareff, B., \& Castets, A. 1997, A\&A, 324, 249

Lefloch, B., Castets, A., Cernicharo, J., \& Loinard, L. 1998, ApJ, 504, L101

Levreault, R. M. 1988, ApJS, 67, 283

Lovas, F. J. 1992, J. Phys. Chem. Ref. Data, 2, 181

Martín-Pintado, J., Bachiller, R., \& Fuente, A. 1992, A\&A, 254,315

Masson, C. R., \& Chernin, L. 1993, ApJ, 414, 230

Matthews, T. J. 1979, A\&A, 75, 345

Nisini, B., Giannini, T., Molinari, S., et al. 1998, in Star Formation with the Infrared Space Observatory, ASP Conf. Ser. 132, ed. J. L. Yun, \& R. Liseau, 256

Nisini, B., Massi, F., Vitali, F., et al. 2001, A\&A, in press

Osterbrock, D. E. 1957, ApJ, 125, 622

Panagia, N. 1973, AJ, 78, 929

Raga, A., \& Cabrit, S. 1993, A\&A, 278, 267

Reipurth, B. 1983, A\&A, 117, 183

Saraceno, P., Ceccarelli, C., Clegg, P., et al. 1996, A\&A, 315, $\mathrm{L} 293$

Saraceno, P., et al. 2001, in preparation

Schwartz, R. D., Gyulbudaghian, A. L., \& Wilking, B. A. 1991, ApJ, 370, 263

Scoville, N. Z., Carlstrom, J. E., Chandler, C. J., et al. 1993, PASP, 105, 1482

Serabyn, E., Güsten, R., \& Mundy, L. 1993, ApJ, 404, 247

Sharpless, S. 1959, ApJS, 4, 257

Shu, F. H., Ruden, S. P., Lada, C. J., \& Lizano, S. 1991, ApJ, 370, L31

Slysh, V. I., Val'tts, I. E., Migenes, V., et al. 1999, ApJ, 526, 236

Sternberg, A., \& Dalgarno, A. 1989, ApJ, 338, 197

Sugitani, K., Tamura, M., \& Ogura, K. 1995, ApJ, 455, L39

Sugitani, K., Fukui, Y., Mizuno, A., \& Ohashi, N. 1989, ApJ, 342, L87

Swade, D. A. 1989, ApJ, 345, 828

Tafalla, M., Myers, P. C., Mardones, D., \& Bachiller, R. 1999, A\&A, 348, 479

Tielens, A. G. G. M., \& Hollenbach, D. J. 1985, ApJ, 291, 722

Tofani, G., Felli, M., Taylor, G. B., \& Hunter, T. R. 1995, A\&AS, 112, 299

Turner, B. E., Chan, K.-W., Green, S., \& Lubowich, D. A. 1992, ApJ, 399, 114

Walker, C. K., Adams, F. C., \& Lada, C. J. 1990, ApJ, 349, 515

Weikard, H., Wouterloot, J. G. A., Castets, A., Winnewisser, G., \& Sugitani, K. 1996, A\&A, 309, 581

White, G. J., Lefloch, B., Fridlund, C. V. M., et al. 1997, A\&A, 323,931

Wild, W. 1995, The $30 \mathrm{~m}$ manual - A handbook for the IRAM $30 \mathrm{~m}$ Telescope, IRAM report

Wilking, B. A., Blackwell, J. H., \& Mundy, L. G. 1989a, AJ, 100, 758

Wilking, B. A., Mundy, L. G., Blackwell, J. H., \& Howe, J. E. 1989b, ApJ, 345, 257

Wilking, B., Mundy, L., McMullin, J., Hezel, T., \& Keene, J. 1993, AJ, 106, 250 